\title{
MicroRNA-146a-5p-modified human umbilical cord mesenchymal stem cells enhance protection against renal injury in diabetic nephropathy through facilitating M2 macrophage polarization by targeting TRAF6
}

\section{Yaqi Zhang}

Wuhan University School of Basic Medical Sciences https://orcid.org/0000-0002-1992-3863

Xi Le

Wuhan University School of Basic Medical Sciences

Shuo Zheng

Wuhan hamilton Biotechnology Co

Ke Zhang

Wuhan University School of Basic Medical Sciences

Jing He

Wuhan University School of Basic Medical Sciences

Mengting Liu

Wuhan hamilton biotechnology Co

Chengshu Tu

Wuhan hamilton Biotechnology Co

Wei Rao

wuhan hamilton biotechnology Co

Hongyuan Du

Wuhan hamilton Biotechnology Co

Yu Ouyang

Wuhan University School of Basic Medical Sciences

Changyong Li

Wuhan University School of Basic Medical Sciences

Dongcheng Wu ( $\square$ bcdcwu@hotmail.com )

Wuhan University School of Basic Medical Sciences https://orcid.org/0000-0002-2989-7291

Research

Keywords: Mesenchymal stem cells, Diabetic nephropathy, microRNAs, Macrophage polarization 
Posted Date: September 8th, 2021

DOl: https://doi.org/10.21203/rs.3.rs-871704/v1

License: (c) (i) This work is licensed under a Creative Commons Attribution 4.0 International License. Read Full License 


\section{Abstract \\ Background}

Diabetic nephropathy (DN) is a severe complication of diabetes mellitus and a common cause of endstage renal disease, but has no approved pharmacotherapy. Mesenchymal stem cells (MSCs) possess potent anti-inflammatory and immunomodulatory properties, which render them an attractive therapeutic tool for tissue damage and inflammation.

\section{Methods}

This study was designed to determine the protective effects and underlying mechanisms of human umbilical cord-derived MSCs (UC-MSCs) on streptozotocin-induced DN. Renal function and histological staining were used to evaluate kidney damage. RNA high-throughput sequencing on rat kidney and UCMSC-derived exosomes was used to identify the critical miRNAs. Co-cultivation of macrophage cell line and UC-MSCs-derived conditional medium was used to assess the involvement of macrophage polarization signaling.

\section{Results}

UC-MSC administration significantly improved renal function, reduced the local and systemic inflammatory cytokine levels, and attenuated inflammatory cell infiltration into the kidney tissue in DN rats. Moreover, UC-MSCs shifted macrophage polarization from a pro-inflammatory M1 to an antiinflammatory M2 phenotype. Mechanistically, miR-146a-5p was significantly downregulated and negatively correlated with renal injury in DN rats as determined through high-throughput RNA sequencing. Importantly, UC-MSCs-derived miR-146a-5p promoted M2 macrophage polarization by inhibiting TRAF6STAT1 signaling pathway. Furthermore, miR-146a-5p modification in UC-MSCs enhanced the efficacy of anti-inflammation and renal function improvement.

\section{Conclusions}

Collectively, our findings demonstrate that UC-MSCs-derived miR-146a-5p have the potential to restore renal function in DN rats through facilitating M2 macrophage polarization by targeting TRAF6. It will pave the way for the use of UC-MSCs for therapeutic delivery of miRNAs targeted at kidney diseases.

\section{Introduction}

Diabetic nephropathy (DN) is one of devastating microvascular complication of diabetes mellitus and the most common cause of end-stage renal disease (ESRD), with $30-40 \%$ mortality [ $1-3]$. DN is characterized by specific renal structure and functional alterations such as glomerular hyperfiltration, 
microalbuminuria, thickening of the glomerular basement membrane, interstitial fibrosis, and hypertrophy of mesangial cells $[4,5]$. Despite current pharmacological treatments, including strategies for optimizing glycemic control and inhibitors of the renin-angiotensin system, these conventional treatments provide incomplete kidney protection [6]. Hence, there is an urgent need for novel therapeutic approaches that efficiently delay the disease progression.

The initiating mechanisms underlying the development and progression of renal injury in DN are not well understood, but current knowledge indicates that its pathogenesis is multifactorial. Compelling and increasing evidence has clearly demonstrated that immunity and inflammation play a paramount role in the pathogenesis of DN. Indeed, DN is associated with both systemic and local renal inflammation with the participation of crucial inflammatory cells such as macrophages [7-11]. In response to various signals, macrophages may undergo classical M1 activation or alternative M2 activation. The M1 phenotype is characterized by the expression of high levels of proinflammatory cytokines, high production of oxygen intermediates, and promotion of Th1 response. In contrast, M2 macrophages are considered to be involved in immunomodulation and promotion of tissue remodeling $[12,13]$.

With the advent of cell therapy, mesenchymal stem cells (MSCs) are considered as the most attractive cell source for regenerative medicine and provide a promising strategy to against DN. MSCs are selfrenewing and multipotent progenitors that can differentiate into a variety of cell types [14]. MSCs can be obtained from various tissues, including bone marrow, skeletal muscle, dental pulp, adipose tissue and umbilical cord [15-17]. Growing evidence in recent years has revealed that MSCs have multiple biological functions, including immunomodulation, anti-inflammation, anti-apoptosis and anti-fibrosis [18]. Because of these characteristics, MSCs have been applied in various diseases, such as respiratory diseases [19], circulatory system disease [20], nervous system disease [21], and kidney disease [22-24]. However, the underlying mechanisms of these beneficial effects are not completely elucidated. Of note, human umbilical cord-derived MSCs (UC-MSCs) are much younger and lower immunogenic, and have a higher yield without ethical issues and invasive procedures. Importantly, UC-MSCs can secrete a wide range of multifunctional factors. Thus human UC-MSCs are considered to be a better choice for clinical applications compared to many other MSCs [25].

MicroRNAs (miRNAs) are a class of noncoding single-stranded small RNA with 20-22 nucleotides in length [26]. miRNAs exert a regulatory effect on a wide range of biological cell processes including cell apoptosis, proliferation, and inflammation by incompletely pairing with the 3 '-untranslated region of the target mRNA [27]. As an endogenous RNA, miRNA maintains stable and conservative in mammals. It has been estimated there more than 2500 mature miRNAs in human genome could regulate expression of gene in physiology and disease [28, 29]. Previous studies have implicated that MSCs-derived exosomes mediate intercellular communications through exchange of proteins, mRNAs, and mostly the miRNAs, which negatively regulate expression of target genes in diverse biological processes [30,31]. However, it remains unclear whether and how UC-MSCs-derived miRNA may regulate macrophage polarization in the pathogenesis of DN. 
In this study, using streptozotocin (STZ)-induced DN rat model and in vitro co-culture experiments, we investigated the efficacy and mechanism of UC-MSCs-based protection against renal injury in DN. Our findings demonstrate that miR-146a-5p mediates the beneficial effects of UC-MSCs on functional recovery in DN through facilitating M2 macrophage polarization by targeting TRAF6-STAT1 signaling pathway.

\section{Material And Method}

\section{Animal experiments}

Eight-week-old adult male Sprague Dawley rats (200-220 g) were obtained from Hubei provincial center for disease control and prevention (Wuhan, China). This study was carried out following the recommendations in the guide for Institutional Animal Care and Use Committee procedures of Hubei Provincial Center for Food and Drug Safety Evaluation (Permit Number: 2020011). After one-week adaptive feeding, rats rendered diabetic were induced by a single intraperitoneal (I.P) injection of STZ (S0130, Sigma, USA), a dose of $60 \mathrm{mg} / \mathrm{kg}$ dissolved in $0.1 \mathrm{M}$ cold citrate buffer ( $\mathrm{pH}$ 4.5). After $24 \mathrm{~h}$, rats were checked for blood glucose, and those with blood glucose more than $16.7 \mathrm{mM}$ for three consecutive days were confirmed as diabetes. Metabolic cages were used to collect urine from diabetes rats for measuring volume and protein concentration after STZ treatment on 4- 6 weeks, and proteinuria $\geq 30$ $\mathrm{mg} / 24 \mathrm{~h}$ was verified as DN. At 12 weeks after STZ or citrate buffer injection, rats were euthanized and blood and urine were collected for renal function detection. Serum creatinine and serum urea nitrogen ware measured with kit according to the manufacturer's instructions (C011-1-1, C013-1-1, Nanjing Jiancheng Bioengineering Insitute, China). BCA kit (P0012S, Beyotime, China) was used to measure the concentration of the protein in urine.

\section{Experimental Design and Treatment Protocol}

Rats were randomly divided into the following groups: (1) normal control group; (2) DN group, (3) UC-MSC treatment group I: DN rats were treated with two injections of $2 \times 10^{6} \mathrm{UC}-\mathrm{MSC}$ through the tail vein at week 7 and 8; (4) UC-MSC treatment group II: DN rats were treated with two injections of $2 \times 10^{6}$ UC-MSCs through the tail vein at week 9 and 10 .

In order to explore the therapeutic effect of miR-146a-5p-modified UC-MSCs on DN, we performed another animal experiment and the UC-MSCs were injected at week 9 and 10. Rats were randomly divided into the following groups: (1) normal control group; (2) normal control plus miR-146a-5p mimic-transfected UCMSC treatment group; (3) normal control plus miR-146a-5p mimic NC-transfected UC-MSC treatment group; (4) DN group; (5) DN plus miR-146a-5p mimic-transfected UC-MSC treatment group; (6) DN plus miR-146a-5p mimic NC-transfected UC-MSC treatment group.

\section{Enzyme-linked immunosorbent assay}


The levels of IL-1 $\beta$ and IL-10 in the kidney tissues and serum from experimental rats were detected following the manufacturer's instructions (CSB-E08055r, SB-E04595r, CUSABIO, China). Cytokine levels in the kidney were presented by ratio of cytokine concentration and albumin concentration.

\section{Histological Analysis}

The kidney obtained from experimental rats was cut longitudinal and fixed with $10 \%$ neutral formalin and finally embedded in paraffin. The paraffin was cut into $5 \mu \mathrm{m}$ thick sections. Hematoxylin and eosin (H\&E), periodic acid-Schiff (PAS) and Masson were used to observe the kidneys' morphological changes. Ly6G immunohistochemical staining was performed with a rabbit anti-Ly6G antibody (BS-20073R, 1:200, Bioss, China). F4/80 immunofluorescence staining was performed with rabbit anti-F4/80 antibody (70076, 1:1000, CST, USA) followed by incubation with CY3-tagged secondary antibodies (A32732, 1:400, Thermo, USA). Finally, DAPI (0100 - 20, SouthernBiotech, USA) was used to stain the nucleus.

For analysis, we used Pannoramic MIDI (3DHISTECH, Hungary) to scan each section under the field of light or fluorescence and circled 10 pieces of $1 \mathrm{~mm}^{2}$ around the section randomly by CaseViewer software. Positive cells in each area were counted, and the average score or positive represented the whole section.

\section{Isolation of exosomes from UC-MSCs and HEK293T}

The conditioned medium of UC-MSCs and HEK293T was collected after 72h. The medium was ultrafiltered to remove the cell debris, and then the concentrated medium was ultracentrifuged to separate the exosomes. The detailed isolation method and identification of exosomes were described in our previous work. ${ }^{[32]}$

\section{Cell culture}

The isolation and identification of UC-MSCs refer to the previous work of our laboratory. ${ }^{\left[{ }^{[3]}\right.}$ UC-MSCs were in serum-free stem cell culture medium (Lonza, MD, Walkersville) at $37^{\circ} \mathrm{C}$ and $5 \% \mathrm{CO}_{2}$ concentration. RAW264.7 and THP-1 were purchased from the China Center for Type Culture Collection (Wuhan China). RAW264.7 cells were cultured in DMEM medium containing 10\% fetal bovine serum (FBS; Hyclone, USA), THP1 were cultured in RPMI 1640 medium with $10 \% \mathrm{FBS}$ at $37^{\circ} \mathrm{C}$ and $5 \% \mathrm{CO}_{2}$ concentration in a humidified atmosphere.

\section{RNA extraction and real-time PCR analysis}

Total RNA (> $200 \mathrm{nt})$ was extracted from renal tissue or cultured cells with Trizol (15596-018, Invitrogen, USA), and small RNA (20 $200 \mathrm{nt}$ ) were extracted by RNAiso for Small RNA (9353A, Takara, China) according to the procedure recommended by the manufacturer. RNA was reversed transcribed into CDNA with a reagent kit (RR047B and 638315, Takara, China). Real-time PCR was performed using SYBR Green reagent (CW0957W, CWBIO, China) on the Real-time PCR System (CFX connect, Bio-Rad, USA). The relative gene and miRNAs expression were determined after normalization to the endogenous housekeeping gene GAPDH and U6 with $2^{-\triangle \Delta C t}$ method. Primer sequences are shown in Table 1. 
Table 1

Primers for qRT-PCR

\begin{tabular}{|c|c|}
\hline gene & sequence $\left(5^{\prime}-3^{\prime}\right)$ \\
\hline r GAPDH-F & TGATTTTGGAGGGATCTCGC \\
\hline r GAPDH-R & ACGGATTTGGTCGTATTGGG \\
\hline rIL-1 $\beta-F$ & CGACAGTGAGGAGAATGACC \\
\hline rIL-1 $\beta-R$ & ACCACTTGTTGGCTTATGTT \\
\hline rIL-6-F & CGGAGAGGAGACTTCACAGA \\
\hline rIL-6-R & GGAGAGCATTGGAAGTTGGG \\
\hline r TNF-a-F & TGCCTCAGCCTCTTCTCATT \\
\hline rTNF-a-R & GTGGGTGAGGAGCACGTAGT \\
\hline r IL-10-F & GTCCTTTCACTTGCCCTCATC \\
\hline r IL-10-R & CAAACTGGTCACAGCTTTCGA \\
\hline riNOS-F & СTCACTGTGGCTGTGGTCACCTA \\
\hline riNOS-R & GGGTCTTCGGGCTTCAGGTTA \\
\hline r ARG1-F & CGGCTTGCGAGATGTGG \\
\hline rARG1-R & TAGCCGGGGTGAATACTGG \\
\hline m GAPDH-F & TGTGATGGGTGTGAACCACG \\
\hline m GAPDH-R & CAGTGAGCTTCCCGTTCACC \\
\hline $\mathrm{m}$ iNOS-F & CAGCTGGGCTGTACAAACCTT \\
\hline $\mathrm{m}$ iNOS-R & CATTGGAAGTGAAGCGTTTCG \\
\hline m MCP1-F & СTСАССTGCTGCTACTСАTTC \\
\hline m MCP1-R & TTACGGCTCAACTTCACATTCA \\
\hline m IL-1 $\beta-F$ & ACGGACCCCAAAAGATGAAG \\
\hline$m$ IL-1 $\beta-R$ & CACGGGAAAGACACAGGTAG \\
\hline m IL-6 -F & TGATGGATGCTACCAAACTGGA \\
\hline m IL-6 -R & TGTGACTCCAGCTTATCTCTTGG \\
\hline m TNF-a-F & СTTCTGTCTACTGAACTTCGGG \\
\hline m TNF-a-R & TGATCTGAGTGTGAGGGTCTG \\
\hline m TRAF6-F & CCTGACGGTAAAGTGCCCAA \\
\hline
\end{tabular}




\begin{tabular}{|c|c|}
\hline gene & sequence $\left(5^{\prime}-3^{\prime}\right)$ \\
\hline m TRAF6-R & ACCTGGCACTTCTGGAAAGG \\
\hline m ARG1-F & GATTATCGGAGCGCCTTTCT \\
\hline m ARG1-R & СCACACTGACTCTTCCATTCTT \\
\hline $\mathrm{m}$ IL-10 -F & GCCAGAGCCACATGCTCCTA \\
\hline$m$ IL-10 -R & GATAAGGCTTGGCAACCCAAGTAA \\
\hline h GAPDH-F & CAAGTGAAATGATGGCTTATTAC \\
\hline h GAPDH-R & СTTTCAACACGCAGGACAGGT \\
\hline h iNOS-F & AGCCCTTTACTTGACCTCCTAA \\
\hline$h$ iNOS-R & CAAGTTCCATCTTTCACCCAC \\
\hline h MCP1-F & GCAGCAAGTGTCCCAAAGAA \\
\hline h MCP1-R & CTGGGGAAAGCTAGGGGAAA \\
\hline h IL-1 $\beta-F$ & AGCCACATCGATCAGACACC \\
\hline h IL-1 $\beta-R$ & GTACTCAGCGCCAGCTCG \\
\hline h IL-6-F & TAATGGGCATTCCTTCTTCT \\
\hline h IL-6-R & TGTCCTAACGCTCATACTTTT \\
\hline h TNF-a-F & GGTGCCTATGTCTCAGCCTCTT \\
\hline h TNF-a-R & GCCATAGAACTGATGAGAGGGAG \\
\hline h TRAF6-F & TTTGGTCTTATGGATTGTCCCC \\
\hline h TRAF6-R & GATTGATGCAGCACAGTTGTC \\
\hline h ARG1-F & CTGTGGGAAAAGCAAGCGAG \\
\hline h ARG1-R & CATGGCCAGAGATGCTTCCA \\
\hline h IL-10-F & GAGGTGATGCCCCAAGCTG \\
\hline h IL-10-R & CACGGCCTTGCTCTTGTTTT \\
\hline
\end{tabular}

\section{RNA high-throughput sequencing analysis}

Tissue RNA was extracted from control and DN kidney, and exosome RNA was extracted from HEK293Tand UC-MSCs-derived exosomes. Samples were submitted in biological triplicate to the MajorBio Platform for quality control, library creation, and high-throughput sequencing. 
After total RNA extraction, purity, concentration, and integrity of RNA were determined by Nanodrop (Thermo Fisher Scientific, USA), and Agilent 2100 Bioanalyzer (Agilent Technologies, USA), respectively. For miRNA sequencing, sequencing libraries were generated using NEB Next Ultra small RNA Sample Library Prep Kit for Illumina (NEB, USA) according to manufacturer's instructions. Finally, PCR products were purified and library quality was assessed on the Agilent Bioanalyzer 2100 system (Agilent Technologies, USA). After quality assessment, sequencing of all libraries was performed by the Illumina Hiseq 2500 platform (Illumina, USA). The raw reads were trimmed and cleaned by removing sequences shorter than $18 \mathrm{nt}$ or longer than $30 \mathrm{nt}$. Simultaneously, Q20, Q30, GC-content, and sequence duplication level of clean data was calculated. All the downstream analyses were based on clean data with high quality. Differential miRNA expression analyses were performed using the DESeq R package (v1.18.0, EMBL Heidelberg, Germany).

\section{Luciferase reporter assay}

The predicted 3'-UTR sequence of TRAF6 interacting with miR-146a-5p and mutated sequences within the predicted target sites were synthesized and inserted into the pmirGLO control vector (E1330, Promega, USA). HEK293T were transfected with $7.5 \mathrm{pmol}$ miR-146a-5p or negative control and $2.5 \mu \mathrm{g}$ of the wildtype or mutant 3'-UTR plasmid by Lipofectamine 2000 (11668027, Invitrogen, USA). After $48 \mathrm{~h}$ of transfection, luciferase activity of cells was measured using a Dual Luciferase Assay Kit (FR201-01, TransGen, China). Renilla luciferase was used to normalize the value of firefly luciferase.

\section{microRNA and siRNA transfection}

Both of the microRNA and small interfering RNA (siRNA) were synthesized by the RIBBIO company in Guangzhou, China. The mimic, inhibitor and their NC of miR-146a-5p were transfected into UC-MSCs at a concentration of $100 \mathrm{nM}$ using lipofectamine RNAiMAX (13778150, Invitrogen, USA) as instructed by the manufacturer's protocols. Both RAW264.7 and THP1 were transfected with siRNA at a concentration of 100 nM using Lipofectamine 2000 (11668019, Invitrogen, USA) as instructed by the manufacturer's protocols. miR-146a-5p mimic, miR-146a-5p inhibitor, and TRAF6 siRNA sequences are shown in Table 2 and Table 3.

Table 2

The sequence of miRNAs

\begin{tabular}{|ll|}
\hline Name & sequence $\left(\mathbf{5}^{\prime} \mathbf{3}^{\prime}\right)$ \\
\hline miR-146a-5p mimic sense & UGAGAA CUGAAUUCCAUGGGUU \\
\hline miR-146a-5p mimic antisense & AACCC AUGGAAUUCAGUUCUCA \\
\hline miR-146a-5p inhibitor sense & AACCCAUGGAAUUCAGUUCUCA \\
\hline miR-146a-5p mimic NC sense & UUUGUACUACACAAAAGUACUG \\
\hline miR-146a-5p mimic NC antisense & AAACAUGAUGUGUUUUCAUGAC \\
\hline miR-146a-5p inhibitor NC sense & UUUGUACUACACAAAAGUACUG \\
\hline
\end{tabular}


Table 3

The target sequence of siRNAs

\begin{tabular}{|ll|}
\hline Name & target sequence (5'-3') \\
\hline si-h-TRAF6_001 & GTAGCGCTGTAACAAAAGA \\
\hline si-h-TRAF6_001 & AGGGTCGCCTTGTAAGACA \\
\hline si-h-TRAF6_001 & CATGCACATTCAGTACTTT \\
\hline si-m-TRAF6_001 & GCCTGCATCATCAAATCCA \\
\hline si-m-TRAF6_002 & TAAGCCAACCAGTTACTT \\
\hline si-m-TRAF6_003 & CATTAAGGATGATACATTA \\
\hline
\end{tabular}

\section{Western blot analysis}

Cells and renal tissues were lysed in ice-cold RIPA Lysis buffer (P0013C, Beyotime, China) supplemented with protease inhibitor cocktail and protein concentration was determined by BCA assay. Proteins were separated by sodium dodecyl sulfate-polyacrylamide gel electrophoresis (SDS-PAGE) and then transferred into a PVDF membrane. Antibodies were used in western blot as follows: anti- $\beta$-actin (600041-Ig, 1:5000, Proteintech, China), anti-Arg1(16001-1-AP, 1:5000, Proteintech, China), anti-iNOS (18985-1-AP, 1:2000, Proteintech, China), anti-TRAF6(abs145675, 1:1000, Absin, China), anti-pSTAT1(abs130924, 1:1000, Absin, China).

\section{Statistics}

Data are expressed as means \pm SD. The two-tailed, Student's t-test was employed for comparisons between two groups, and one-way ANOVA were performed for comparisons of data with more than two groups. $\mathrm{P}<0.05$ was considered statistically significant. In vitro experiments were assessed in at least three independent experiments. All analyses were carried out by GraphPad Prism 5.0.

\section{Results}

\section{UC-MSC administration ameliorates renal injury in DN rats}

To explore the therapeutic effect of UC-MSCs, we established a STZ-induced DN model of rats. The experimental design was shown in Fig. 1a, and the difference between the two treatment groups was that UC-MSCs were transplanted at different stages of DN. We analyzed renal function and pathological changes among control rats, DN rats, and UC-MSC-treated DN rats. Compared with DN rats, UC-MSCtreated DN rats showed a significant decrease in serum creatinine (CRE), serum urea nitrogen (BUN) and 24h total urinary protein (Fig. 1b). Moreover, UC-MSC administration attenuated tubular dilatation, massive accumulation of inflammatory cells in the interstitial area, renal glomerular hypertrophy and sclerosis, and renal interstitial fibrosis in the DN kidney (Fig. 1c-1e). These results indicate that UC-MSCs 
ameliorate renal injury in DN rats, and transplantation of UC-MSCs at different stages of DN has a consistent therapeutic effect.

\section{UC-MSC administration inhibits renal inflammation in DN rats}

To investigate whether UC-MSCs modulate the inflammatory response in the DN rats, we determined the levels of pro- and anti-inflammatory cytokines and quantified inflammatory cell infiltration in the DN rats with or without UC-MSC treatment. Unlike in DN group which showed elevated mRNA levels of proinflammatory IL-1 $\beta$, IL- 6 and TNF-a, UC-MSC treatment in rats significantly reduced the expression of proinflammatory cytokines but augmented anti-inflammatory IL-10 expression (Fig. 2a). Consistent with this data, decreased IL-1 $\beta$ production and increased IL-10 production (Fig. 2b and 2c) were observed in kidneys and serum from UC-MSC-treated DN rats, as compared with untreated controls. Moreover, UCMSC administration resulted in a significant decreased accumulation of $\mathrm{Ly}^{6} \mathrm{G}^{+}$neutrophils (Fig. $2 \mathrm{~d}$ ) and F4/80 $0^{+}$macrophages (Fig. 2e), as determined by immunohistochemistry and immunofluorescence staining. These results indicate that UC-MSC administration reduces systemic and local renal inflammation in DN rats.

\section{UC-MSCs shift macrophage polarization toward an M2 phenotype in vivo and in vitro}

Since macrophage $\mathrm{M} 1$ activation is implicated in the pathogenesis of chronic inflammatory diseases including $\mathrm{DN}^{[34]}$, we next determined whether UC-MSCs may regulate macrophage polarization in DN rats. The results showed that administration of UC-MSCs increased arginase1 (ARG1) expression, an M2 macrophage marker, but decreased inducible nitric oxide synthase (iNOS) expression, an M1 macrophage marker, at the mRNA and protein levels (Fig. 3a, 3b). Moreover, using double immunofluorescence staining, we found that UC-MSC administration enhanced M2 macrophage ARG1 expression (Fig. 3c).

Next, we used UC-MSC-conditioned medium (UC-MSC-CM) to treat the macrophage cell line RAW264.7 in response to stimulation with LPS $(100 \mathrm{ng} / \mathrm{ml})$ and IFN-y $(30 \mathrm{ng} / \mathrm{ml})$. Clearly, UC-MSC-CM treatment resulted in significant decrease in the expressions of iNOS, IL-1 $\beta$, TNF- $\alpha$, and TRAF6, but increase in ARG1 and IL-10 expression in LPS/IFN-y-stimulated RAW264.7, as compared with untreated controls (Fig. 3d, $3 e)$. To further demonstrate the effect of UC-MSCs on macrophage polarization, we used human macrophage cell line THP1. Similarly, UC-MSC-CM treatment markedly reduced iNOS, IL-1 $\beta$, TNF-a, TRAF6 and p-STAT1 expression, but increased ARG1 and IL-10 expression in LPS/IFN- $\gamma$-stimulated THP1 (SuppI Fig. 1). Taken together, these results suggest UC-MSCs regulate macrophage polarization and function during inflammatory response via a paracrine mechanism.

\section{High-throughput sequencing analysis of miRNA profile in the DN kidney and UC-MSCs}

miRNAs have been implicated as the key regulators of different molecules involved in the DN network [35]. To explore the communication between miRNAs and macrophage polarization in DN, we examined the miRNAs in kidney tissues of normal and DN rats using high-throughput sequencing. Moreover, we measured the expression levels of various miRNAs between exosomes derived from HEK293T and UC- 
MSCs. Analysis of differentially expressed miRNAs revealed that miR-146a-5p was significantly downregulated in the DN kidney and abundant in UC-MSC-derived exosomes (Fig. 4a, 4b). Further validation of miR-146a-5p expression in the kidney by qRT-PCR confirmed the expression of miR-146a-5p was downregulated in the DN rats and restored in the UC-MSC-treated DN rats (Fig. 4c). We also found that miR146a-5p expression was significantly increased in LPS/IFN-y-stimulated UC-MSCs, as compared with unstimulated controls. (Fig. 4d). More importantly, the expression of miR-146a-5p in the kidney was negatively correlated with the concentration of Cre and BUN in serum (Fig. 4e, 4f). These results reveal the potential function and candidate biomarker attributes of miR-146a-5p in DN, and suggest that UC-MSCderived miR-146a-5p may play an important role in protection against $\mathrm{DN}$.

\section{UC-MSCs-derived miR-146a-5p targets TRAF6 and facilitates M2 macrophage polarization}

To identify the mechanism how miR-146a-5p regulates macrophage polarization during $\mathrm{DN}$, we performed bioinformatics analyses according to TargetScan and microRNA.org. The web-based prediction software for targets of miRNAs, TRAF6 3'-UTR region contains the putative binding sequence of miR-146a-5p, could be a potential target gene of miR-146a-5p (Fig. 5a). Using the dual-luciferase assay, we observed that miR-146a-5p mimics significantly suppressed luciferase activity when wide type (WT) 3'UTR of TRAF6 was inserted downstream of the luciferase report plasmid, as compared with mutant 3'UTR (Fig. 5b).

Moreover, we transfected miR-146a-5p mimic, miR-146a-5p inhibitor, and their negative control (NC) into UC-MSCs, and collected UC-MSC-CM to treat LPS/IFN- $\gamma$-stimulated RAW264.7. As expected, transfection with miR-146a-5p mimic obviously increased miR-146a-5p expression in UC-MSCs, as compared with control UC-MSCs (Fig. 5c). We also observed that CM derived from miR-146a-5p mimic-transfected UCMSCs markedly decreased the expressions of iNOS, IL-1 $\beta$, TNF-a, TRAF6 and p-STAT1, but significantly increased ARG1 and IL-10 expressions compared to control UC-MSCs-CM. However, CM derived from miR146a-5p inhibitor-transfected UC-MSCs exerted the opposite results (Fig. 5d, 5e). Similar results were observed in human macrophage cell line THP1. Transfection with miR-146a-5p mimic leaded to promote M2 macrophage polarization, and transfection with miR-146a-5p inhibitor resulted in facilitating M1 macrophage polarization in THP1 cells (Supplementary Fig. 1). Together, these results demonstrate that miR-146a-5p contributes to UC-MSCs-mediated M2 macrophage polarization.

\section{TRAF6 is required for UC-MSC-miR-146a-5p-mediated M2 macrophage polarization.}

To further elucidate whether miR-146a-5p regulated macrophage polarization by targeting TRAF6, we used TRAF6 siRNA to knockdown TRAF6 in macrophage cell line and cocultured with miR-146a-5p inhibitor transfected-UC-MSCs. The knockdown efficiency of TRAF6 in RAW264.7 and THP1 was detected by qRT-PCR (Suppl Fig. 2a, 2c) and western blot analyze (Suppl Fig. 2b, 2c). We found that TRAF6 downregulation resulted in decreased expressions of iNOS, IL-1 $\beta$, TNF- $\alpha$ and p-STAT1, and elevated ARG1 expression in RAW264.7 cells treated with CM derived from miR-146a-5p inhibitor-transfected UC-MSCs (Fig. 6a, 6b). Similar results were observed in THP1, indicating the elevated anti-inflammatory factors and reduced pro-inflammatory factors in the presence of miR-146a-5p inhibitor when TRAF6 was silenced 
(Suppl Fig. 2e, 2f). These results demonstrate that TRAF6 plays essential role in miR-146a-5p-regulated macrophage polarization.

\section{MiR-146a-5p-modified UC-MSCs enhance the efficacy of anti-inflammation and renal function improvement in DN rats.}

To further confirm the regulatory role of miR-146a-5p in macrophage polarization in vivo, we transfected miR-146a-5p mimic or miR-146a-5p mimic NC into UC-MSCs, and transplanted the modified-UC-MSCs into DN rats via the tail vein. Subsequently, we determined the levels of inflammatory cytokines, quantified inflammatory cell infiltration and M1/M2 macrophage marker expression in the experimental rats. The results showed that administration of miR-146a-5p mimic-transfected UC-MSCs in DN rats obviously reduced Cre, BUN and $24 \mathrm{~h}$ total urinary protein compared to miR-146a-5p mimic NC-transfected UC-MSCs (Fig. 7a). Moreover, miR-146a-5p mimic-transfected UC-MSC administration further attenuated kidney pathological damage (Suppl Fig. 3). As compared with miR-146a-5p mimic NC-transfected UCMSC administration in DN rats, decreased IL-1ßand increased IL-10 production (Fig. 7b and 7c) were observed in kidney tissues and serum from miR-146a-5p mimic-transfected UC-MSCs-treated DN rats. miR-146a-5p mimic-transfected UC-MSC administration in DN rats also resulted in a significant

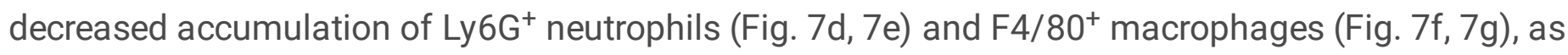
determined by immunohistochemistry and immunofluorescence staining. Moreover, using double immunofluorescence staining, we found that miR-146a-5p mimic-transfected UC-MSC administration enhanced M2 macrophage ARG1 expression in DN rats (Fig. 8a). Notably, western blot analysis indicated the expression of iNOS, TRAF6 and p-STAT1 were significantly decreased, but ARG1 expression was increased in miR-146a-5p mimic-transfected UC-MSC-treated DN rats (Fig. 8b). Collectively, these results reveal that miR-146a-5p-modified UC-MSCs enhance the efficacy of renal function improvement and regulation of macrophage polarization in DN.

\section{Discussion}

DN is the most prevalent chronic renal disease and the major cause of end-stage renal failure. Inflammation is considered the key mediators of the development and progression of renal injury in DN $[9,10]$. In this study, we demonstrated that UC-MSC administration significantly improved renal function of DN rats. Importantly, UC-MSCs facilitated M2 macrophage differentiation, accompanied by reduced systemic and local renal inflammation in DN rats. We further identified that miR-146a-5p was dramatically decreased and negatively correlated with renal injury in DN rats. Furthermore, UC-MSCderived miR-146a-5p shifted M2 macrophage polarization by targeting TRAF6-STAT1 signaling pathway. Finally, we proved miR-146a-5p-modified UC-MSCs enhanced the efficacy of anti-inflammation and renal function improvement in DN rats.

Data from other groups as well as our previous studies revealed that MSCs represent promising cellbased therapeutics for tissue injury and disease, including chronic kidney disease [33, 36, 37]. However, the exact molecular mechanisms for MSC-based therapy for DN have not been fully elucidated. It is well 
documented that MSCs may provide a means for recapitulating multiple mechanisms to repair tissue injury, including immunomodulation, antioxidant, autophagy, and anti-fibrosis [38-40]. Notably, the potent immunomodulatory and anti-inflammatory properties of MSCs are currently the focus of intensive studies in graft enhancement, tissue protection, and regenerative medicine [41]. Multiple lines of evidence are presented to show that inflammation is a key pathogenic factor during the pathogenesis of DN, and that imbalance of M1/M2 macrophages plays a central role in inflammation [13]. Here we discovered that UC-MSCs reduced systemic and local renal inflammation, at least in part, through facilitating macrophage polarization from a pro-inflammatory M1 to an anti-inflammatory M2 phenotype in the DN kidney. It should be noted that although there are studies exploring the clinical effects of MSCs on DN, the timing and therapeutic window for cell therapy remains elusive. In this study, DN rats were treated with two injections of UC-MSCs at week 7 and 8, or week 9 and 10, respectively. Our results showed that administration of UC-MSCs at these two different stages of DN has similar protection against renal injury and inflammation, demonstrating the therapeutic efficacy of UC-MSC injection in the early or late phase of DN.

miRNAs carried by circulating exosomes have been widely proved as significant contributors to tissue homeostasis and feasible therapeutics for various diseases [42, 43]. Indeed, miRNAs have recently emerged as important regulators of DN. However, the role of miRNAs in the regulation of inflammation during DN is not fully understood. Importantly, MSCs have been demonstrated to be a safe and effective delivery vehicle for therapeutic miRNA treatment, due to their ability to specifically target immune disorder, inflammation and fibrosis $[44,45]$. In this study, through high-throughput RNA sequencing, we found that UC-MSC-exosomes had a specific miRNA abundance signature that was different from that of HEK293T-exosomes. In particular, we identified that UC-MSC-derived miR-146a-5p played a critical role in macrophage polarization during DN. Previous studies reported that miR-146a-5p was shown to be involved in the regulation of inflammatory response [46, 47]. Here, our findings demonstrate that UCMSCs exert a protective effect on DN through delivery of miR-146a-5p, resulting in suppression of inflammation. These studies suggest that miR-146a-5p might act as an important anti-inflammatory noncoding RNA modulator of DN and other inflammatory diseases. More importantly, our study revealed a novel role of exosomal miRNA in UC-MSC-mediated therapy in DN. However, further work is needed to evaluate the therapeutic efficacy of UC-MSC-derived exosomes in DN rats.

TRAF6 is an adaptor protein that mediates a wide array of protein-protein interactions. In the context of the immune system, TRAF6-mediated signals have proven critical for the development, homeostasis, and activation of innate immune cells, including macrophages [48]. More recent works have shown that TRAF6 plays a crucial role in the induction of inflammatory responses via activation of the nuclear factor kappa B (NF-KB) pathway $[49,50]$. In addition, STAT proteins are identified as a family of latent cytoplasmic transcription factors and play a critical role in transducing signals from various cytokines to achieve distinct transcriptional outcomes [51]. Recently, emerging evidence suggests that STATs play important roles in macrophage polarization [52]. Among the family members, evidence suggested that IFN- $\gamma$ activates JAK-STAT1 signaling and promotes STAT1 phosphorylation, which leads to the M1 macrophage polarization [53] Luu and colleagues [54] also demonstrated cross-talk between the TLR and 
JAK/STAT signaling pathways with direct recruitment of STAT1 by TRAF6. In keeping these studies, our data suggested that TRAF6-STAT1 signaling was involved in renal inflammation in DN rats. More importantly, we identified that UC-MSCs-derived miR-146a-5p targeted the TRAF6-STAT1 pathway to suppress kidney inflammation and restore renal function through facilitating M2 macrophage polarization.

With the ability of immunomodulation and anti-inflammation, MSC-based therapy has been applied in various immune- and inflammation-mediated diseases [55]. However, a number of phase III clinical trials of MSC immunotherapy were unable to meet the primary endpoints because of the low immunoregulatory efficacy of engrafted cells [56]. In the quest to circumvent these challenges, several modification techniques have been applied to improve the therapeutic efficacy of MSCs. For instance, hepatocyte growth factor or vascular endothelial growth factor overexpressing MSCs maximized MSCbased myocardial salvage after acute myocardial infarction [57]. CXCR4 receptor overexpression in MSCs improved MSC homing and facilitated treatment of acute lung injury in rats [58]. In this study, we demonstrated that miR-146a-5p-modified UC-MSCs promoted M2 macrophage polarization and enhanced protection against renal injury in DN rats, which further proved the key role of miR-146a-5p in macrophage polarization during $\mathrm{DN}$.

\section{Conclusion}

we identify a role of miR146a-5p/TRAF6 signaling in controlling macrophage polarization in UC-MSCmediated immune regulation. Our findings demonstrate a potential beneficial effect of UC-MSC administration on the pathophysiology of STZ-induced DN. Importantly, the present study suggests that miR146a-5p-modifed UC-MSCs enhance protection against renal injury in DN through facilitating M2 macrophage polarization by targeting TRAF6-STAT1 signaling, which may offer new therapeutic approaches for DN currently lacking effective treatment. Further studies are required to optimize dose, timing, and duration of UC-MSCs and to delineate the multiple molecular mechanisms underlying UCMSC protection against DN.

\section{Abbreviations}

DN: Diabetic nephropathy; ESRD: end-stage renal disease; MSCs: mesenchymal stem cells; UC-MSCs: human umbilical cord-derived MSCs; miRNAs: MicroRNAs; STZ: streptozotocin; H\&E: Hematoxylin and eosin; PAS: Periodic Acid-Schiff; CRE: creatinine; BUN: urea nitrogen; IL-6: Interleukin 6; IL-1ß: Interleukin 1 $\beta$; TNF-a: Tumor necrosis factor-a; IL-10: Interleukin 10; ARG1: arginase1; iNOS: inducible nitric oxide synthase; UC-MSC-CM: UC-MSC-conditioned medium; NC: negative control; RAW264.7: Mouse mononuclear macrophage leukemia cells; THP1: Human myeloid leukemia monocytes; HEK293T: Human embryonic kidney cells; TRAF6: TNF receptor associated factor 6; STAT1: Signal transducer and activator of transcription 1

\section{Declarations}




\section{Acknowledgement}

Not applicable

\section{Author contributions}

Y.Z. and X.L. performed in vivo and in vitro experiments, and data analysis; S.Z. and K.Z. performed in vivo experiments; J.H. provided the results of high-throughput sequencing in exosomes; M.L., C.T., W.R., H.D. and Y.O. participated in scientific discussion; and C.L. and D.W. contributed to the study concept, research design, data analysis, and C.L. wrote the manuscript.

\section{Funding}

This work was supported by grants from Wuhan Municipal Science and Technology Bureau (2019030703011513, 2020020601012210), 2020 Special Project for the Central Government to Guide the Development of Local Science and Technology ("Hundred Cities and Hundred Parks "Action), and the Innovation and Entrepreneurship Leading Team Project in Zengcheng District, Guangzhou (202001002).

\section{Availability of data and materials}

All data generated and/or analyzed during this study are included in this published article.

\section{Ethics approval and consent to participate}

The care and use of animals were approved by the Committee of Animal Care and Use of Hubei Provincial Center for Food and Drug Safety Evaluation and Animal Experiment (Permit No: 2020011). The use of the human umbilical cord tissue from a healthy donor who gave birth and signed informed consent in Renmin Hospital was supported by the Institutional Ethics Review Board of Renmin Hospital of Wuhan University (Permit Number: WDRY2019-G001).

\section{Consent for publication}

Not applicable.

\section{Competing interests}

The authors declare that they have no competing interests.

\section{References}

1. Bell S, Fletcher EH, Brady I, Looker HC, Levin D, Joss N, et al. End-stage renal disease and survival in people with diabetes: a national database linkage study. QJM. 2015;108(2):127-34.

2. Heerspink HJL, Parving HH, Andress DL, Bakris G, Correa-Rotter R, Hou FF, et al. Atrasentan and renal events in patients with type 2 diabetes and chronic kidney disease (SONAR): a double-blind, 
randomised, placebo-controlled trial. Lancet. 2019;393(10184):1937-47.

3. Duran-Salgado MB, Rubio-Guerra AF. Diabetic nephropathy and inflammation. World J Diabetes. 2014;5(3):393-8.

4. Fineberg D, Jandeleit-Dahm KA, Cooper ME. Diabetic nephropathy: diagnosis and treatment. Nat Rev Endocrinol. 2013;9(12):713-23.

5. Sierra-Mondragon E, Molina-Jijon E, Namorado-Tonix C, Rodriguez-Munoz R, Pedraza-Chaverri J, Reyes JL. All-trans retinoic acid ameliorates inflammatory response mediated by TLR4/NF-kappaB during initiation of diabetic nephropathy. J Nutr Biochem. 2018;60:47-60.

6. Fried LF, Emanuele N, Zhang JH, Brophy M, Conner TA, Duckworth W, et al. Combined angiotensin inhibition for the treatment of diabetic nephropathy. N Engl J Med. 2013;369(20):1892-903.

7. Matoba K, Takeda Y, Nagai Y, Kawanami D, Utsunomiya K, Nishimura R. Unraveling the Role of Inflammation in the Pathogenesis of Diabetic Kidney Disease. Int J Mol Sci. 2019;20(14).

8. Moreno JA, Gomez-Guerrero C, Mas S, Sanz AB, Lorenzo O, Ruiz-Ortega M, et al. Targeting inflammation in diabetic nephropathy: a tale of hope. Expert Opin Investig Drugs. 2018;27(11):91730.

9. Lim AK, Tesch GH. Inflammation in diabetic nephropathy. Mediators Inflamm. 2012;2012:146154.

10. Wada J, Makino H. Inflammation and the pathogenesis of diabetic nephropathy. Clin Sci (Lond). 2013;124(3):139-52.

11. Pichler R, Afkarian M, Dieter BP, Tuttle KR. Immunity and inflammation in diabetic kidney disease: translating mechanisms to biomarkers and treatment targets. Am J Physiol Renal Physiol. 2017;312(4):F716-F31.

12. Wynn TA, Vannella KM. Macrophages in Tissue Repair, Regeneration, and Fibrosis. Immunity. 2016;44(3):450-62.

13. Sica A, Mantovani A. Macrophage plasticity and polarization: in vivo veritas. J Clin Invest. 2012;122(3):787-95.

14. Jiang $Y$, Jahagirdar BN, Reinhardt RL, Schwartz RE, Keene CD, Ortiz-Gonzalez XR, et al. Pluripotency of mesenchymal stem cells derived from adult marrow. Nature. 2002;418(6893):41-9.

15. Orbay H, Tobita M, Mizuno H. Mesenchymal stem cells isolated from adipose and other tissues: basic biological properties and clinical applications. Stem Cells Int. 2012;2012:461718.

16. Venkat P, Shen Y, Chopp M, Chen J. Cell-based and pharmacological neurorestorative therapies for ischemic stroke. Neuropharmacology. 2018;134(Pt B):310-22.

17. Mennan C, Brown S, McCarthy H, Mavrogonatou E, Kletsas D, Garcia J, et al. Mesenchymal stromal cells derived from whole human umbilical cord exhibit similar properties to those derived from Wharton's jelly and bone marrow. FEBS Open Bio. 2016;6(11):1054-66.

18. Peired AJ, Sisti A, Romagnani P. Mesenchymal Stem Cell-Based Therapy for Kidney Disease: A Review of Clinical Evidence. Stem Cells Int. 2016;2016:4798639. 
19. Liu A, Zhang X, He H, Zhou L, Naito Y, Sugita S, et al. Therapeutic potential of mesenchymal stem/stromal cell-derived secretome and vesicles for lung injury and disease. Expert Opin Biol Ther. 2020;20(2):125-40.

20. Chen Q, Liu Y, Ding X, Li Q, Qiu F, Wang M, et al. Bone marrow mesenchymal stem cell-secreted exosomes carrying microRNA-125b protect against myocardial ischemia reperfusion injury via targeting SIRT7. Mol Cell Biochem. 2020;465(1-2):103-14.

21. Xiong L, Sun L, Zhang Y, Peng J, Yan J, Liu X. Exosomes from Bone Marrow Mesenchymal Stem Cells Can Alleviate Early Brain Injury After Subarachnoid Hemorrhage Through miRNA129-5p-HMGB1 Pathway. Stem Cells Dev. 2020;29(4):212-21.

22. Eliopoulos N, Zhao J, Forner K, Birman E, Young YK, Bouchentouf M. Erythropoietin gene-enhanced marrow mesenchymal stromal cells decrease cisplatin-induced kidney injury and improve survival of allogeneic mice. Mol Ther. 2011;19(11):2072-83.

23. Selim RE, Ahmed HH, Abd-Allah SH, Sabry GM, Hassan RE, Khalil WKB, et al. Mesenchymal Stem Cells: a Promising Therapeutic Tool for Acute Kidney Injury. Appl Biochem Biotechnol. 2019;189(1):284-304.

24. Klein J, Gonzalez J, Miravete M, Caubet C, Chaaya R, Decramer S, et al. Congenital ureteropelvic junction obstruction: human disease and animal models. Int J Exp Pathol. 2011;92(3):168-92.

25. Wang M, Yuan Q, Xie L. Mesenchymal Stem Cell-Based Immunomodulation: Properties and Clinical Application. Stem Cells Int. 2018;2018:3057624.

26. Bartel DP. MicroRNAs: genomics, biogenesis, mechanism, and function. Cell. 2004;116(2):281-97.

27. Ha M, Kim VN. Regulation of microRNA biogenesis. Nat Rev Mol Cell Biol. 2014;15(8):509-24.

28. Bartel DP. MicroRNAs: target recognition and regulatory functions. Cell. 2009;136(2):215-33.

29. Wojciechowska A, Braniewska A, Kozar-Kaminska K. MicroRNA in cardiovascular biology and disease. Adv Clin Exp Med. 2017;26(5):865-74.

30. Chen Y, Ding H, Wei M, Zha W, Guan S, Liu N, et al. MSC-Secreted Exosomal H19 Promotes Trophoblast Cell Invasion and Migration by Downregulating let-7b and Upregulating F0X01. Mol Ther Nucleic Acids. 2020;19:1237-49.

31. Sharma A. Role of stem cell derived exosomes in tumor biology. Int J Cancer. 2018;142(6):1086-92.

32. Deng T, He J, Yao Q, Wu L, Xue L, Wu M, et al. Human Umbilical Cord Mesenchymal Stem Cells Improve Ovarian Function in Chemotherapy-Induced Premature Ovarian Failure Mice Through Inhibiting Apoptosis and Inflammation via a Paracrine Mechanism. Reprod Sci. 2021;28(6):1718-32.

33. Xiang E, Han B, Zhang Q, Rao W, Wang Z, Chang C, et al. Human umbilical cord-derived mesenchymal stem cells prevent the progression of early diabetic nephropathy through inhibiting inflammation and fibrosis. Stem Cell Res Ther. 2020;11(1):336.

34. Olefsky JM, Glass CK. Macrophages, inflammation, and insulin resistance. Annu Rev Physiol. 2010;72:219-46. 
35. Dewanjee S, Bhattacharjee N. MicroRNA: A new generation therapeutic target in diabetic nephropathy. Biochem Pharmacol. 2018;155:32-47.

36. Wu Y, Zhang C, Guo R, Wu D, Shi J, Li L, et al. Mesenchymal Stem Cells: An Overview of Their Potential in Cell-Based Therapy for Diabetic Nephropathy. Stem Cells Int. 2021;2021:6620811.

37. Chen L, Xiang E, Li C, Han B, Zhang Q, Rao W, et al. Umbilical Cord-Derived Mesenchymal Stem Cells Ameliorate Nephrocyte Injury and Proteinuria in a Diabetic Nephropathy Rat Model. J Diabetes Res. 2020;2020:8035853.

38. Li Y, Liu J, Liao G, Zhang J, Chen Y, Li L, et al. Early intervention with mesenchymal stem cells prevents nephropathy in diabetic rats by ameliorating the inflammatory microenvironment. Int $\mathrm{J}$ Mol Med. 2018;41(5):2629-39.

39. Ebrahim N, Ahmed IA, Hussien NI, Dessouky AA, Farid AS, Elshazly AM, et al. Mesenchymal Stem Cell-Derived Exosomes Ameliorated Diabetic Nephropathy by Autophagy Induction through the mTOR Signaling Pathway. Cells. 2018;7(12).

40. Duni A, Liakopoulos V, Roumeliotis S, Peschos D, Dounousi E. Oxidative Stress in the Pathogenesis and Evolution of Chronic Kidney Disease: Untangling Ariadne's Thread. Int J Mol Sci. 2019;20(15).

41. Shi Y, Wang Y, Li Q, Liu K, Hou J, Shao C, et al. Immunoregulatory mechanisms of mesenchymal stem and stromal cells in inflammatory diseases. Nat Rev Nephrol. 2018;14(8):493-507.

42. Valadi H, Ekstrom K, Bossios A, Sjostrand M, Lee JJ, Lotvall JO. Exosome-mediated transfer of mRNAs and microRNAs is a novel mechanism of genetic exchange between cells. Nat Cell Biol. 2007;9(6):654-9.

43. Chandrasekaran K, Karolina DS, Sepramaniam S, Armugam A, Wintour EM, Bertram JF, et al. Role of microRNAs in kidney homeostasis and disease. Kidney Int. 2012;81(7):617-27.

44. Chen C, Wang D, Moshaverinia A, Liu D, Kou X, Yu W, et al. Mesenchymal stem cell transplantation in tight-skin mice identifies miR-151-5p as a therapeutic target for systemic sclerosis. Cell Res. 2017;27(4):559-77.

45. Wang B, Yao K, Huuskes BM, Shen HH, Zhuang J, Godson C, et al. Mesenchymal Stem Cells Deliver Exogenous MicroRNA-let7c via Exosomes to Attenuate Renal Fibrosis. Mol Ther. 2016;24(7):1290301.

46. Bhatt K, Lanting LL, Jia Y, Yadav S, Reddy MA, Magilnick N, et al. Anti-Inflammatory Role of MicroRNA-146a in the Pathogenesis of Diabetic Nephropathy. J Am Soc Nephrol. 2016;27(8):227788.

47. Fang SB, Zhang HY, Wang C, He BX, Liu XQ, Meng XC, et al. Small extracellular vesicles derived from human mesenchymal stromal cells prevent group 2 innate lymphoid cell-dominant allergic airway inflammation through delivery of miR-146a-5p. J Extracell Vesicles. 2020;9(1):1723260.

48. Walsh MC, Lee J, Choi Y. Tumor necrosis factor receptor- associated factor 6 (TRAF6) regulation of development, function, and homeostasis of the immune system. Immunol Rev. 2015;266(1):72-92.

49. Xie C, Zhang LZ, Chen ZL, Zhong WJ, Fang JH, Zhu Y, et al. A hMTR4-PDIA3P1-miR-125/124-TRAF6 Regulatory Axis and Its Function in NF kappa B Signaling and Chemoresistance. Hepatology. 
2020;71(5):1660-77.

50. Min Y, Kim MJ, Lee S, Chun E, Lee KY. Inhibition of TRAF6 ubiquitin-ligase activity by PRDX1 leads to inhibition of NFKB activation and autophagy activation. Autophagy. 2018;14(8):1347-58.

51. O'Shea JJ, Murray PJ. Cytokine signaling modules in inflammatory responses. Immunity. 2008;28(4):477-87.

52. Ding N, Wang Y, Dou C, Liu F, Guan G, Wei K, et al. Physalin D regulates macrophage M1/M2 polarization via the STAT1/6 pathway. J Cell Physiol. 2019;234(6):8788-96.

53. Miklossy G, Hilliard TS, Turkson J. Therapeutic modulators of STAT signalling for human diseases. Nat Rev Drug Discov. 2013;12(8):611-29.

54. Luu K, Greenhill CJ, Majoros A, Decker T, Jenkins BJ, Mansell A. STAT1 plays a role in TLR signal transduction and inflammatory responses. Immunol Cell Biol. 2014;92(9):761-9.

55. Wang LT, Ting CH, Yen ML, Liu KJ, Sytwu HK, Wu KK, et al. Human mesenchymal stem cells (MSCs) for treatment towards immune- and inflammation-mediated diseases: review of current clinical trials. J Biomed Sci. 2016;23(1):76.

56. Karp JM, Leng Teo GS. Mesenchymal stem cell homing: the devil is in the details. Cell Stem Cell. 2009;4(3):206-16.

57. Deuse T, Peter C, Fedak PW, Doyle T, Reichenspurner H, Zimmermann WH, et al. Hepatocyte growth factor or vascular endothelial growth factor gene transfer maximizes mesenchymal stem cell-based myocardial salvage after acute myocardial infarction. Circulation. 2009;120(11 Suppl):247-54.

58. Yang JX, Zhang N, Wang HW, Gao P, Yang QP, Wen QP. CXCR4 receptor overexpression in mesenchymal stem cells facilitates treatment of acute lung injury in rats. $J$ Biol Chem. 2015;290(4):1994-2006.

\section{Figures}


a

$\mathrm{STZ}(60 \mathrm{mg} / \mathrm{kg})$, i.p.

Treatment I hUC-MSCs $2 \times 10^{6}$, twice, i.v. Sacrifice, sampling

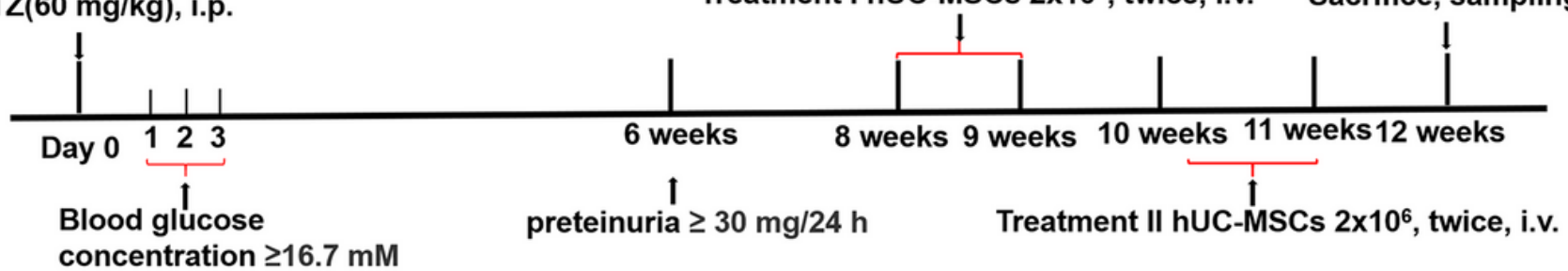

b
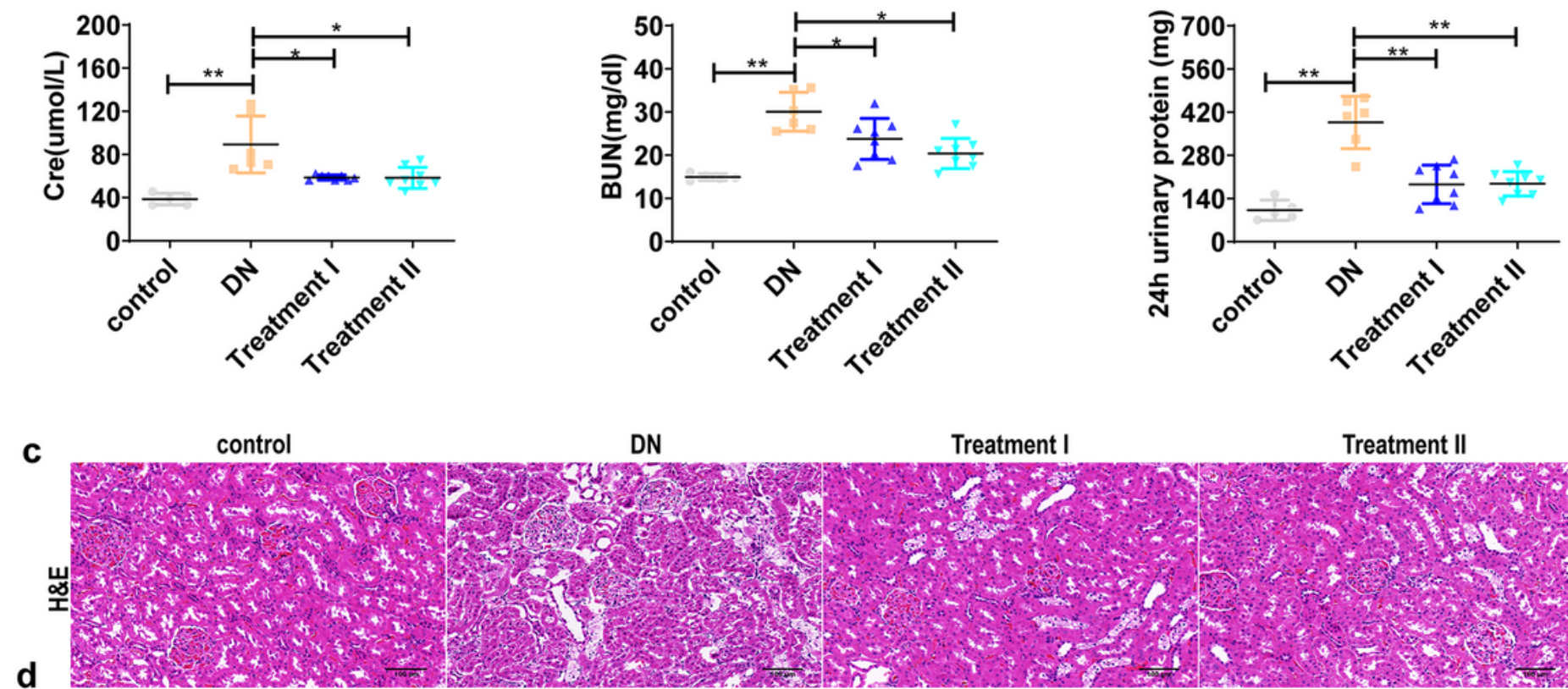

DN Treatment I

Treatment II

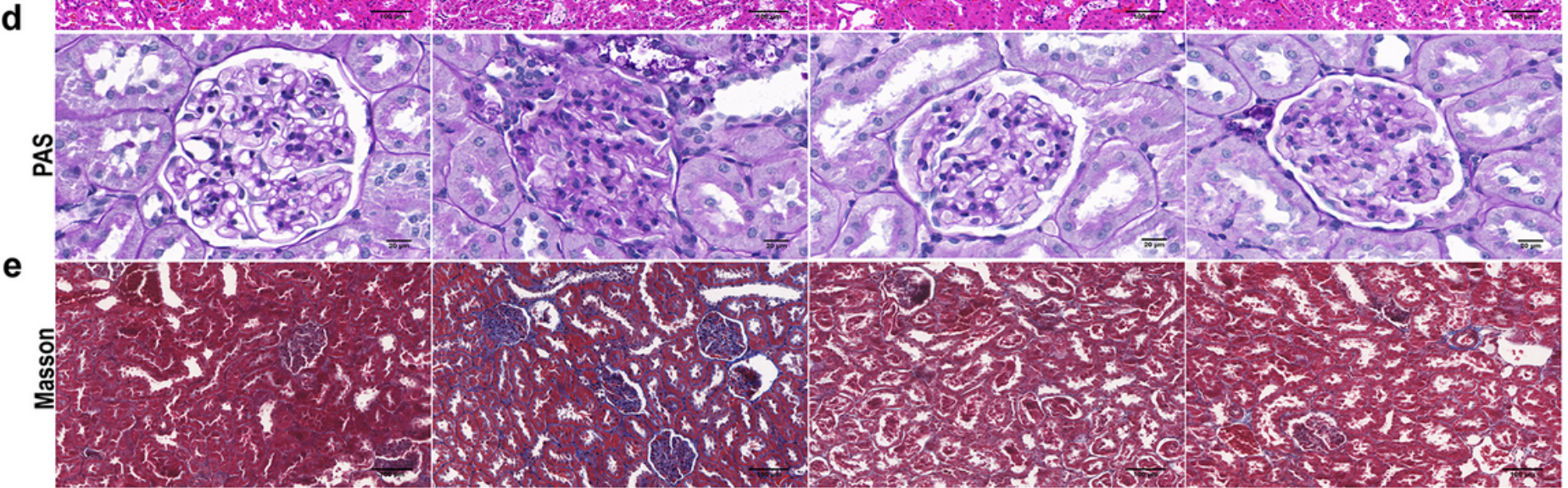

Figure 1

UC-MSC administration ameliorates renal injury in DN rats. Rats were exposed to $60 \mathrm{mg} / \mathrm{kg} \mathrm{STZ} \mathrm{by}$ intraperitoneal to induce DN model. UC-MSCs $(2 \times 106 / 500 \mu \mathrm{l})$ were administrated via the tail veil to assess the therapeutic effect. a Flowchart and timetable of rat treatment from day 0 to week 12. b Renal function in DN rats with or without UC-MSC administration was determined by measuring serum creatinine, serum urea nitrogen, $24 \mathrm{~h}$ total urine protein and creatinine clearance rate at week $12, \mathrm{n}=5-8$ rats/group. H\&E staining (c), PAS staining (d) and Masson staining (e) in the kidneys to observe histological changes, $n=3$ rats/group, Scale bar: $100 \mu \mathrm{m}$ in H\&E and Masson, Scale bar: $20 \mu \mathrm{m}$ in PAS. 
Data presented as mean \pm SD of individuals included in each group. $p$ values were calculated using Student's t-test. * $P<0.05$, ** $P<0.01$.

a
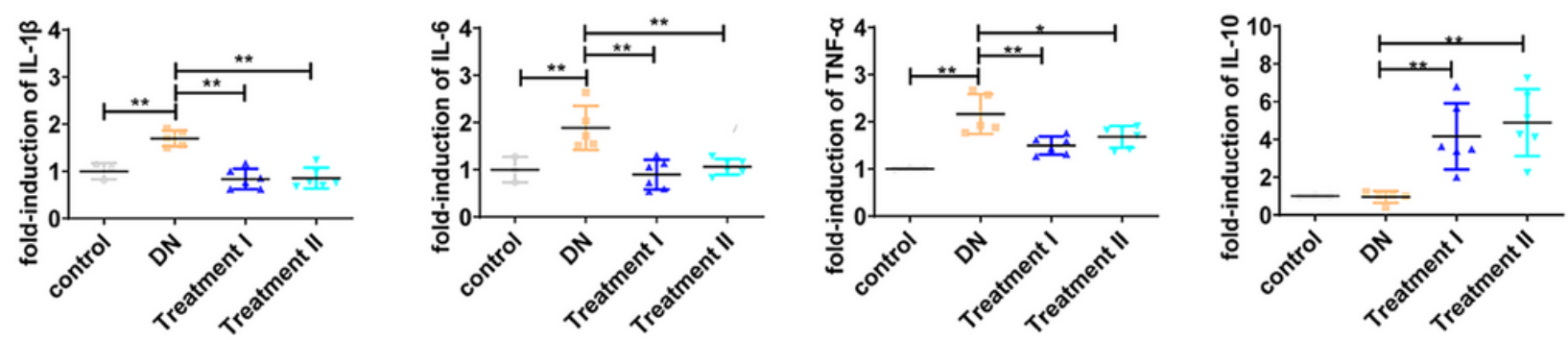

b

Serum

c

Kidney tissue
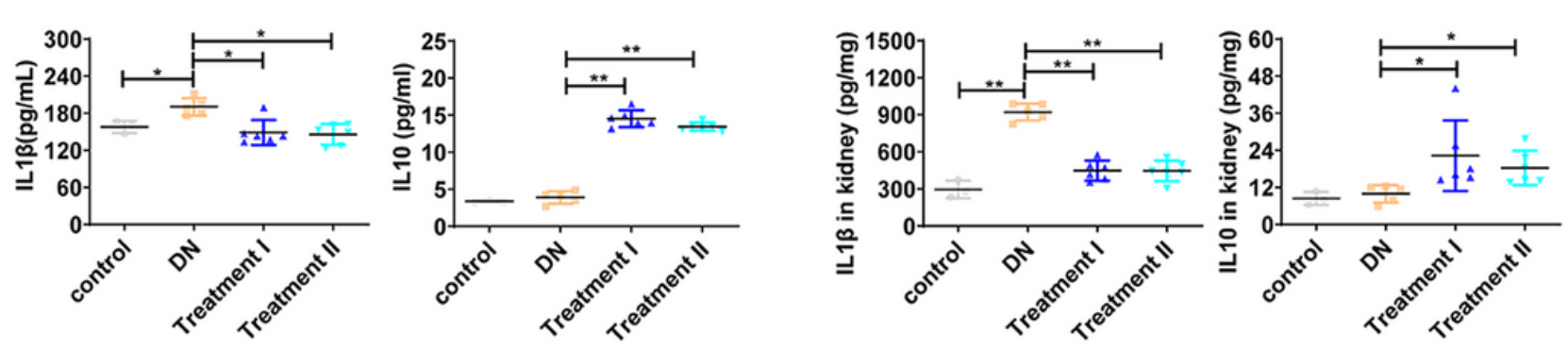

d

DN

Treatment I

Treatment II
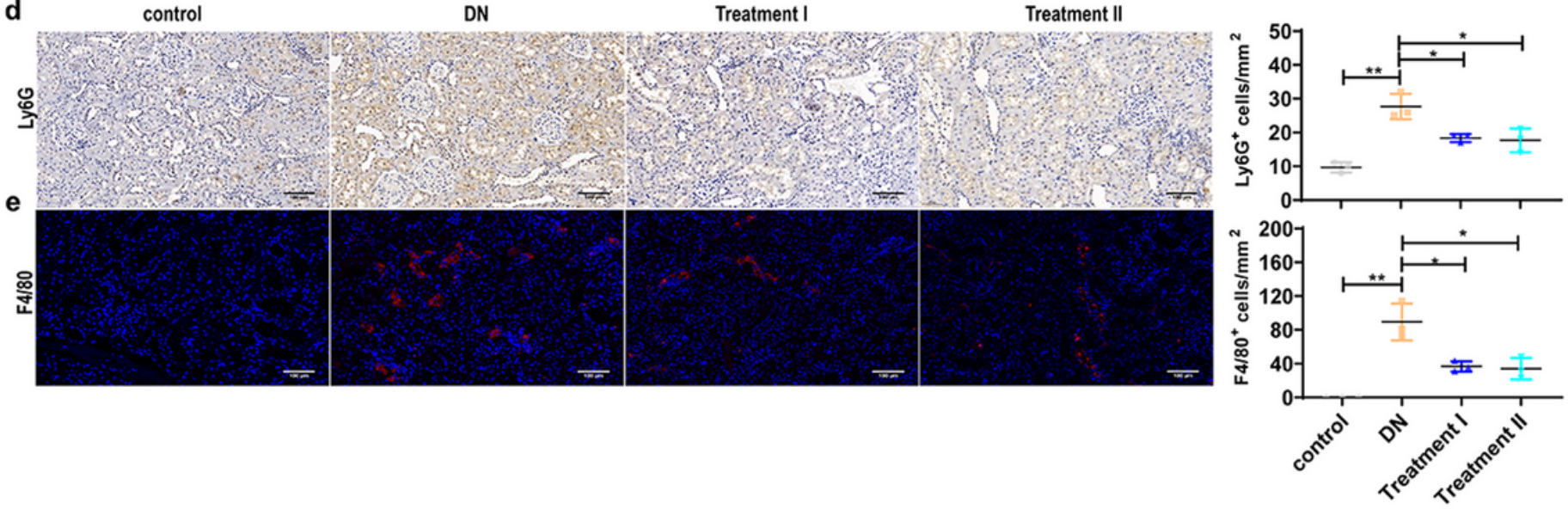

Figure 2

UC-MSC administration inhibits renal inflammation in DN rats. a Relative mRNA expression of IL-6, IL-1 $\beta$, TNF-a, and IL-10 in the kidney from DN rats with or without UC-MSC administration. b Concentration of IL$1 \beta$ in the kidney and serum as measured by ELISA. c Concentration of IL-10 in the kidney and serum as measured by ELISA ( $n=5-8$ rats/group). $d$ Infiltrating neutrophils in the kidney as represented by Ly6G immunohistochemistry staining. e Infiltrating macrophages in the kidney as represented by F4/80 immunofluorescence staining. $n=3$ rats/group, Scale bar: $100 \mu \mathrm{m}$. Data presented as mean \pm SD of individuals included in each group. $p$ values were calculated using Student's t-test. * $P<0.05, * \star P<0.01$. 


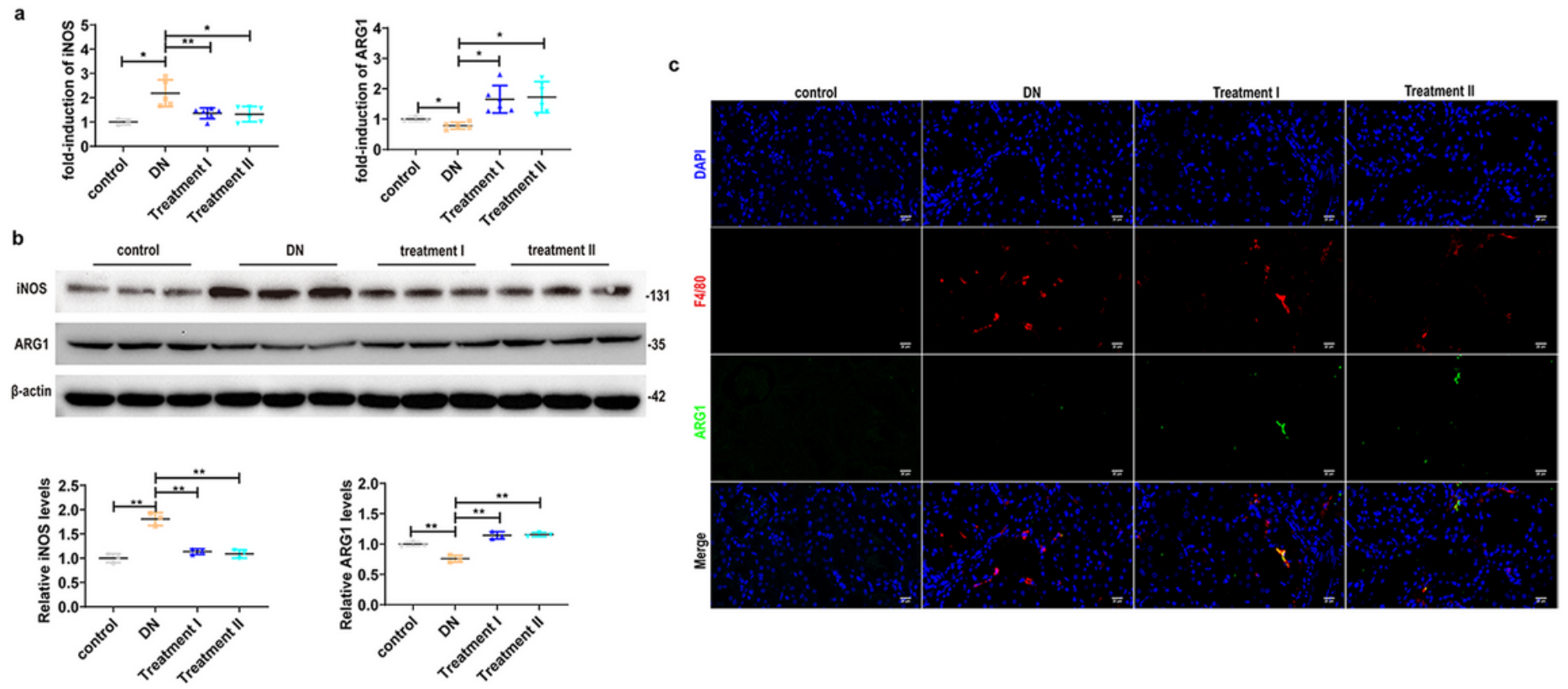

d
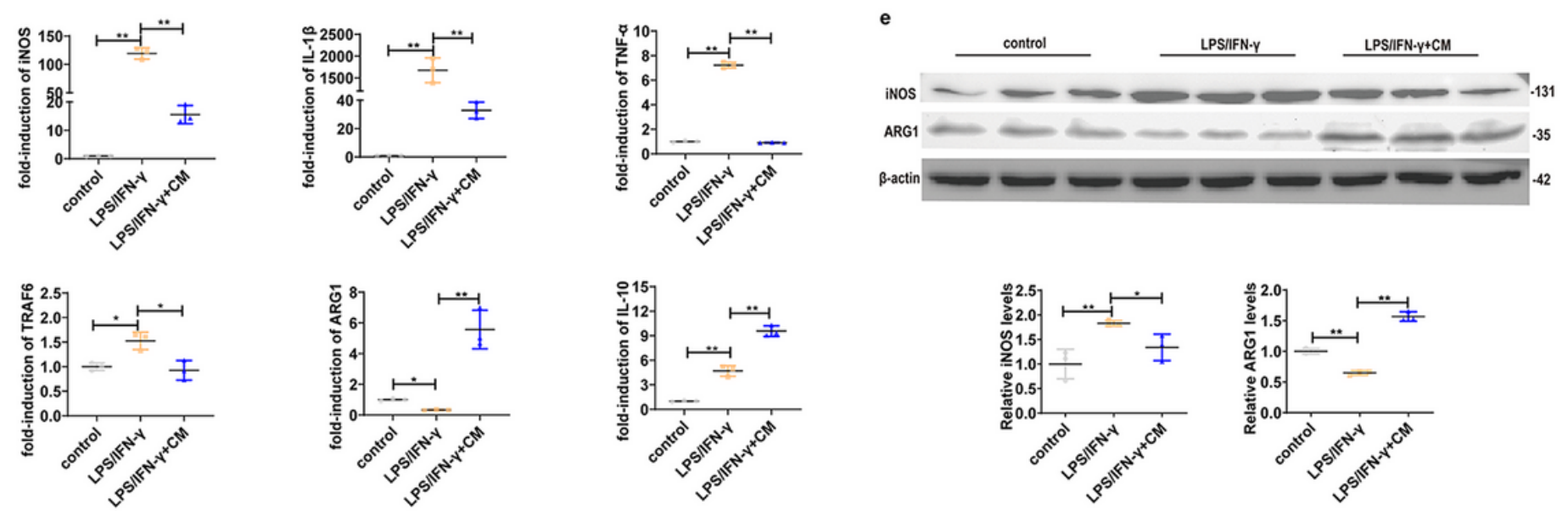

Figure 3

UC-MSCs shift macrophage polarization toward an M2 phenotype. a Relative mRNA expression of iNOS and ARG1 in the kidney from DN rats with or without UC-MSC administration, $n=5-8$ rats/group. b Relative protein expression of iNOS and ARG1 in the kidney, $n=3$ rats/group. c Representative immunofluorescence staining for the macrophage marker F4/80 (red) and ARG1 (green) colocalization in the kidney from DN rats with or without UC-MSC administration, Scale bar: $20 \mu \mathrm{m}$. d RAW264.7 cell were stimulated with LPS/IFN- $\gamma$ and treated with UC-MSC- conditioned medium (UC-MSC-CM) to explore the effect of UC-MSC-CM on regulation of macrophage polarization in vitro. Relative mRNA expression of inflammatory cytokines and macrophage markers including iNOS, IL-1 $\beta$, TNF-a, TRAF6, ARG1, and IL-10. e Relative protein expression and semi-quantitative analysis of M1/M2 macrophage markers (iNOS and $A R G 1)$ in RAW264.7. Data presented as mean \pm SD of individuals included in each group. Results in vitro are representative of three independent experiments. $p$ values were calculated using paired Student's $t$ test. $* \mathrm{P}<0.05, * * \mathrm{P}<0.01$. 
a

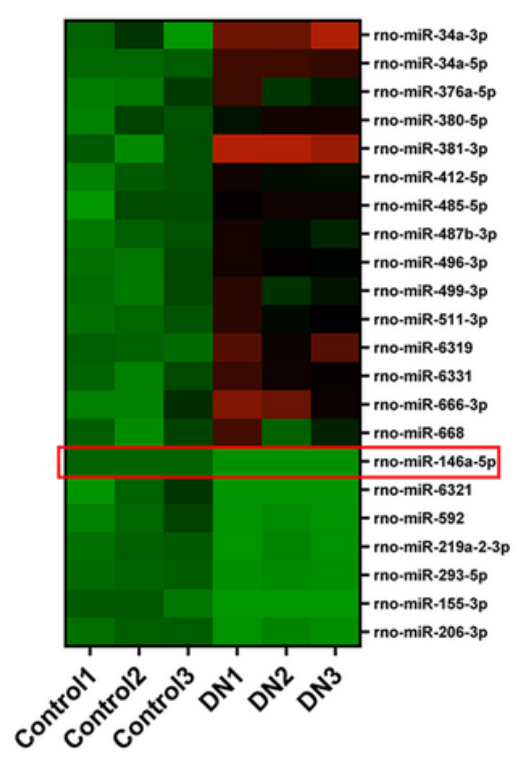

b

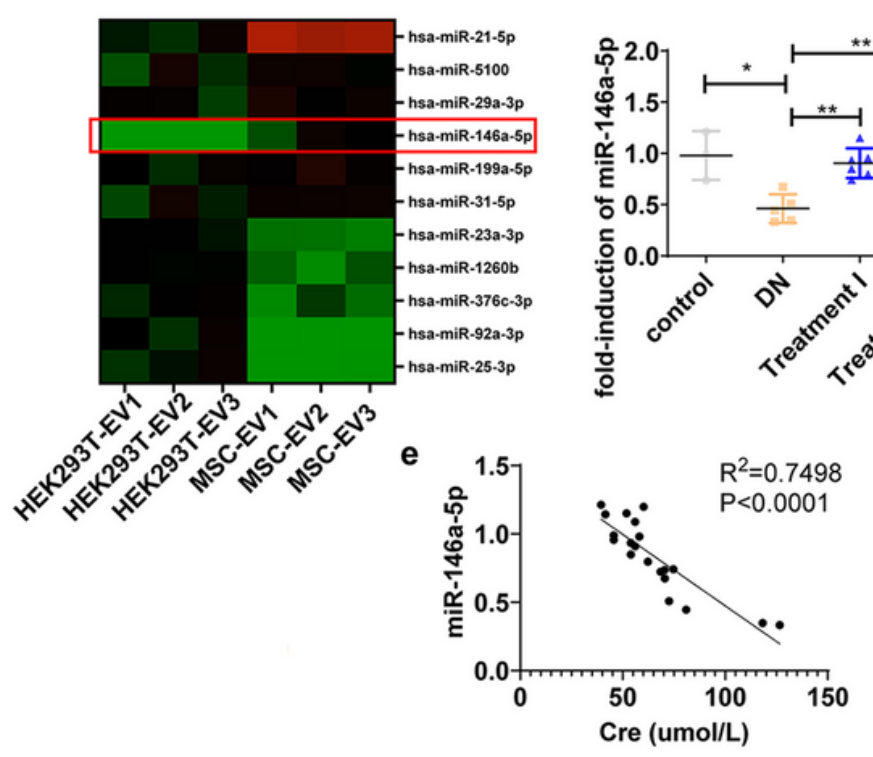

d
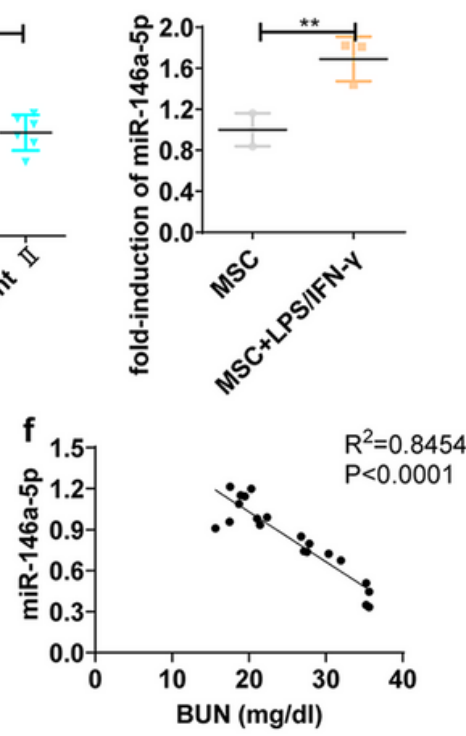

Figure 4

High-throughput sequencing analysis of miRNA profile in the DN kidney and UC-MSCs. miRNAs highthroughput sequencing was performed to identify specific miRNAs which were abnormal expression in the DN kidney and abundant in UC-MSCs derived exosomes. a The heatmap of down- or up-regulated miRNAs identified by RNA high-throughput sequencing in the control and DN kidney, $n=3$ rats/group. $b$ The heatmap of down- or up-regulated miRNAs identified by high-throughput sequencing in the HEK293Tand UC-MSC-derived exosomes, $n=3$ samples/group. $c$ The expression of miR-146a-5p in the kidney from DN rats with or without UC-MSC administration, $n=5-8$ rats/group. $d$ The expression of miR-146a-5p in UC-MSCs with or without LPS $(100 \mathrm{ng} / \mathrm{ml}) / \mathrm{IFN}-\gamma(30 \mathrm{ng} / \mathrm{ml})$ stimulation. e Correlation analysis of miR$146 a-5 p$ and serum Cre in rats. $f$ Correlation analysis of miR-146a-5p and serum BUN in rats, $n=20$ rats. Data presented as mean \pm SD of individuals included in each group. Results in vitro are representative of three independent experiments. $p$ values were calculated using Student's t-test. * $P<0.05,{ }^{*} P<0.01$. 
a

miR-146a-5p: 3 '-UUGGGUACCUUAAGUCAAGAGU-5

TRAF6 473-480 WT: 5'- TCTAGAAAGTTGAGTTCTCATTTTTTTAACCTGATTA-3'

TRAF6 473-480 mut: 5'- TCTAGAAAGTTGCTTAAGAGTTTTTTTAACCTGATTA-3' b

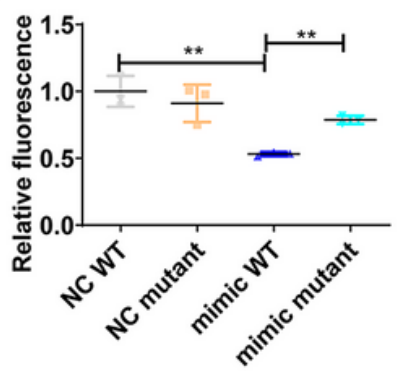

C

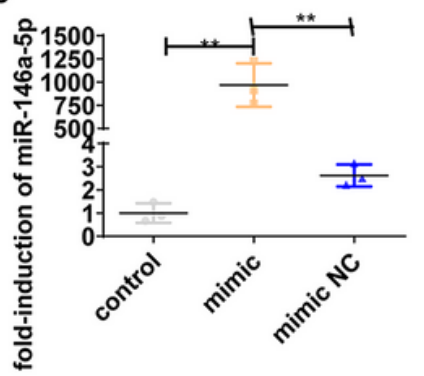

d
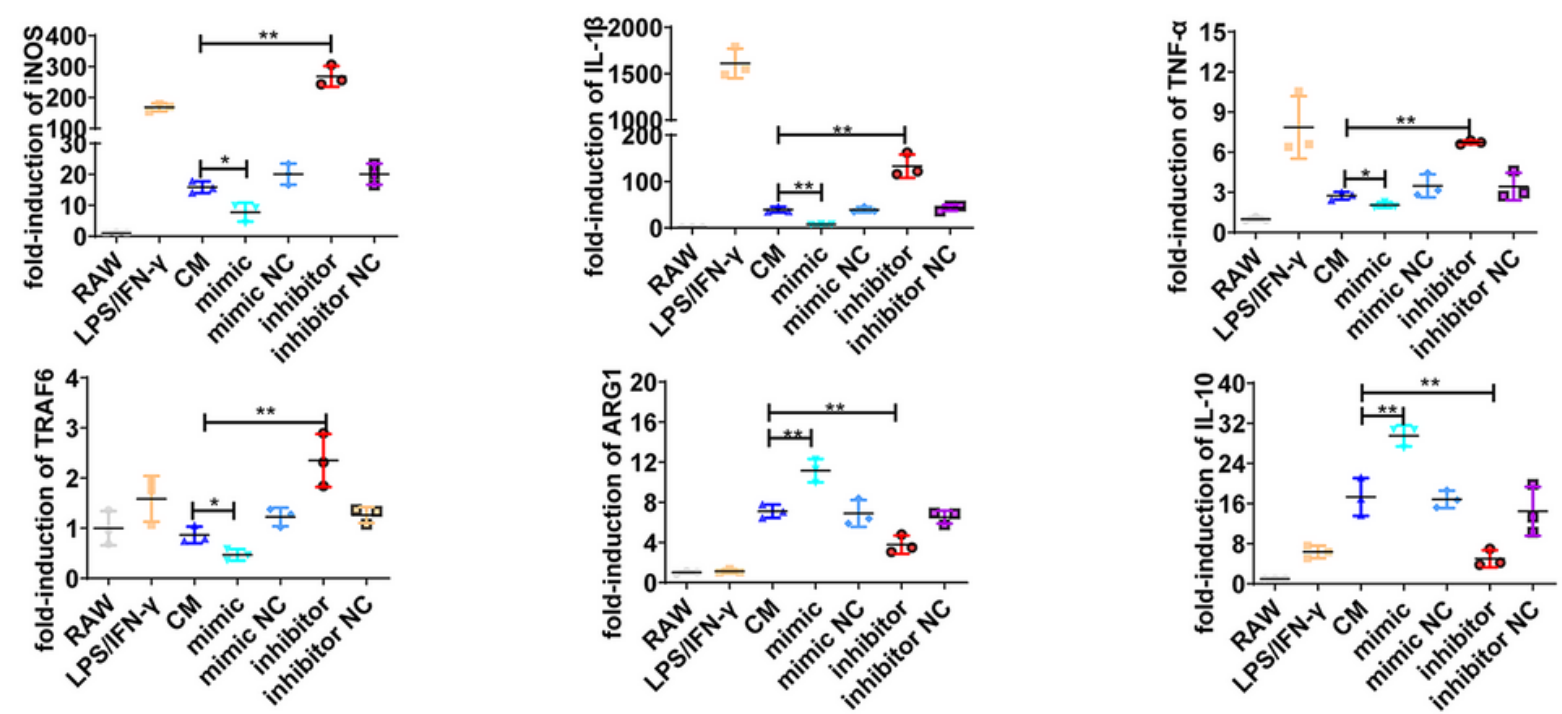

e
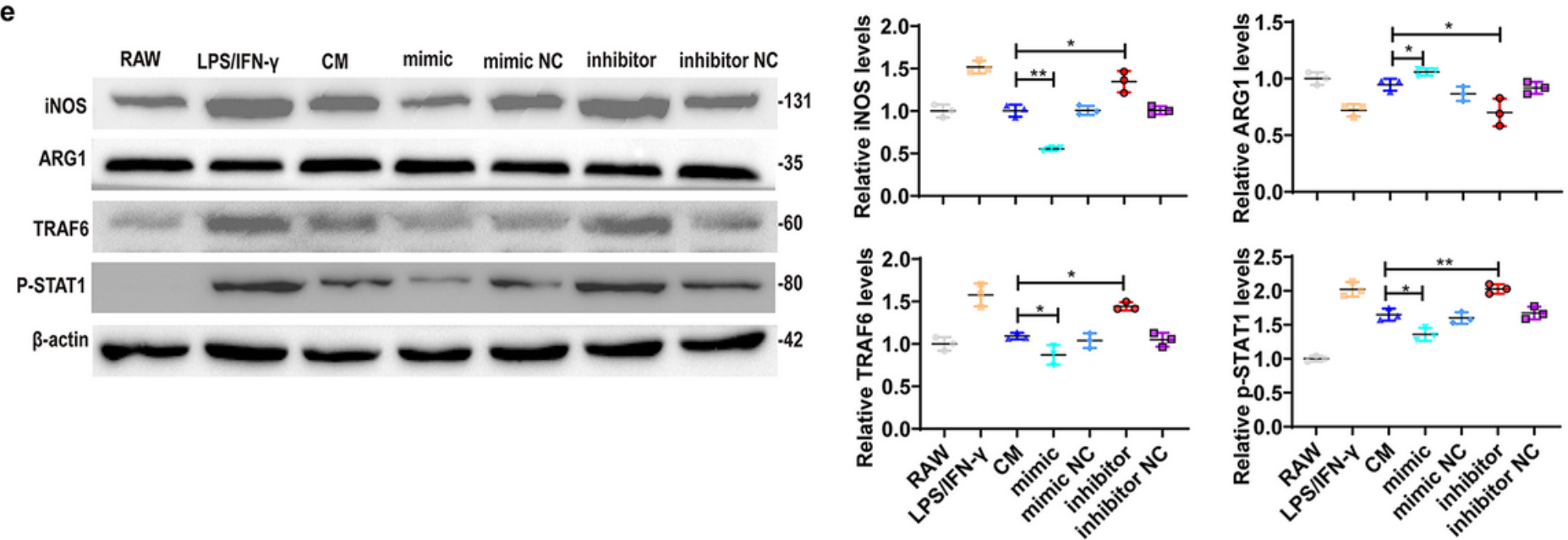

Figure 5

UC-MSC-derived miR-146a-5p targets TRAF6 and facilitates M2 macrophage polarization in RAW264.7. a TargetScan predicted miR-146a-5p binding sites in the 3'UTR at 473-480 sites of TRAF6 and the corresponding designed mutation site. b Luciferase activity was measured in HEK293T cells were cotransfected with miR-146a-5p mimic or miR-146a-5p NC and luciferase reporter plasmids containing wildtype or mutant. c The expression of miR-146a-5p in control UC-MSCs, miR-146a-5p mimic transfected UCMSCs and miR-146a-5p mimic NC transfected UC-MSCs. The miR-146a-5p mimic, miR-146a-5p inhibitor 
and their negative controls were transfected into UC-MSCs, and the CM was collected to treat RAW264.7. d Relative mRNA expression of inflammatory cytokines and M1/M2 macrophage markers including iNOS, IL-1 $\beta$, TNF- $\alpha$, TRAF6, ARG1, and IL-10 in the control RAW264.7, LPS/IFN- - -stimulated RAW264.7 and LPS/IFN-ץ-stimulated RAW264.7 treated with the indicated CM. e Relative protein expression and semiquantitative analysis of iNOS, ARG1, TRAF6, and p-STAT1 in RAW264.7. Data presented as mean \pm SD in each group. Results in vitro are representative of three independent experiments. $p$ values were calculated using Student's t-test. * $\mathrm{P}<0.05, * \star P<0.01$.

a
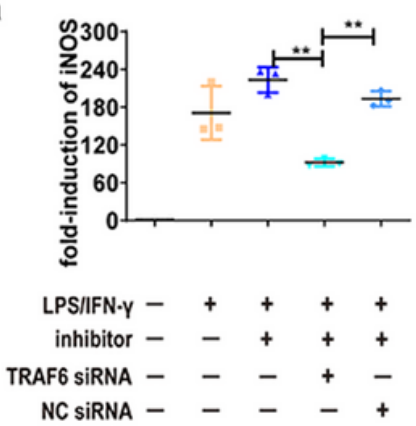
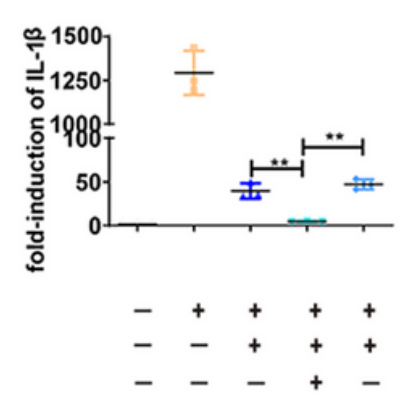
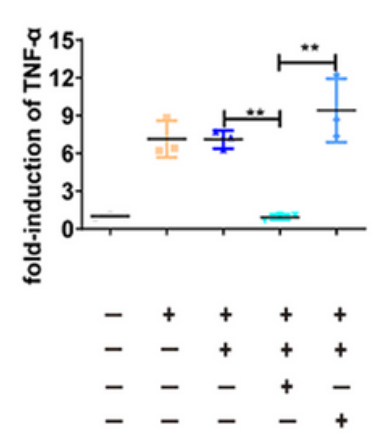
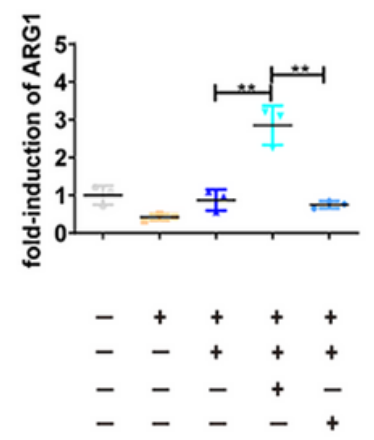

b
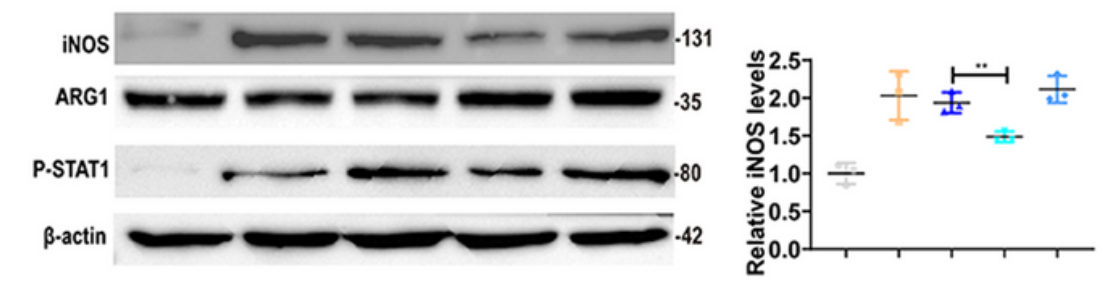

$\begin{array}{rllll}\text { LPSIIFN.Y - } & + & + & + & + \\ \text { inhibitor - } & - & + & + & + \\ \text { TRAF6 siRNA - } & - & - & + & - \\ \text { NC siRNA - } & - & - & - & +\end{array}$
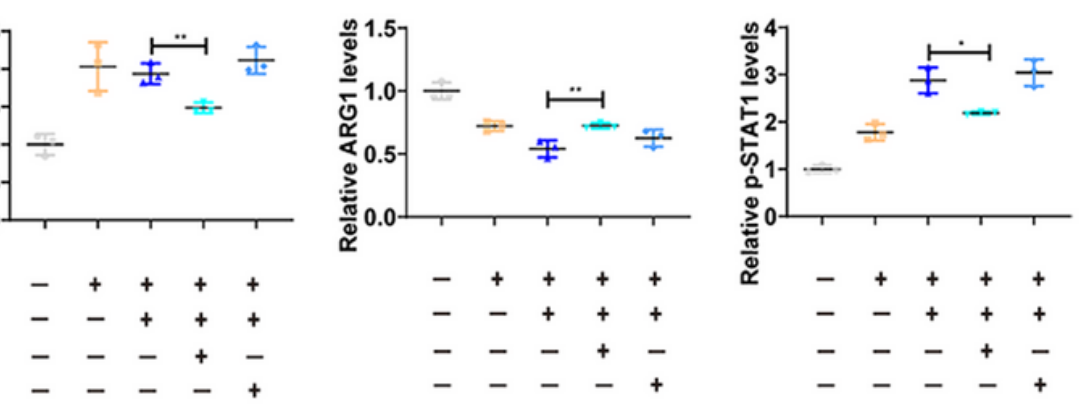

\section{Figure 6}

TRAF6 is required for UC-MSCs-derived miR-146a-5p-mediated M2 macrophage polarization. RAW264.7 cells were transfected with TRAF6 siRNA or negative control (NC) siRNA, and then treated with CM derived from miR-146a-5p inhibitor transfected-UC-MSCs. a Relative mRNA expression of inflammatory cytokines and M1/M2 macrophage markers including iNOS, IL-1 $\beta$, TNF- $a$, and ARG1 in RAW264.7. b Relative protein expression and semi-quantitative analysis of INOS, ARG1, and p-STAT1 in RAW264.7. Data presented as mean $\pm S D$ in each group. Results in vitro are representative of three independent experiments. $p$ values were calculated using Student's t-test. * $P<0.05, * * P<0.01$. 
a

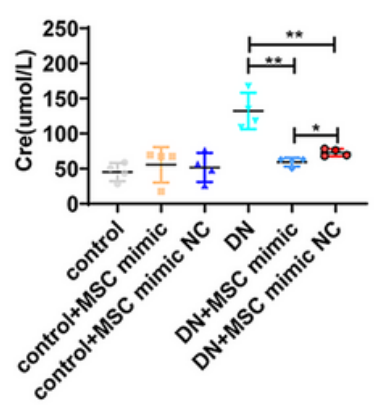

b

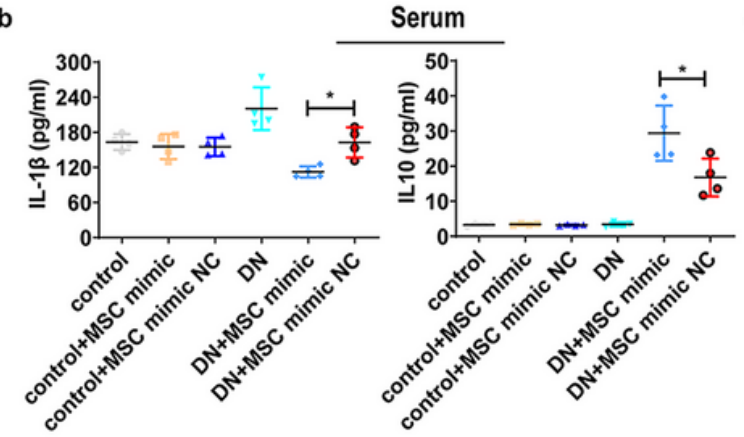

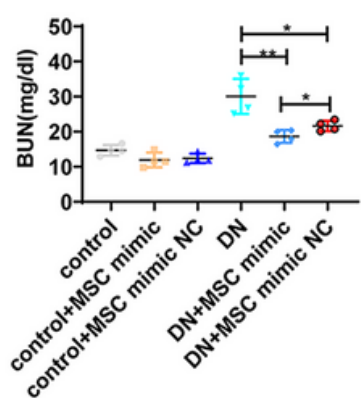

c

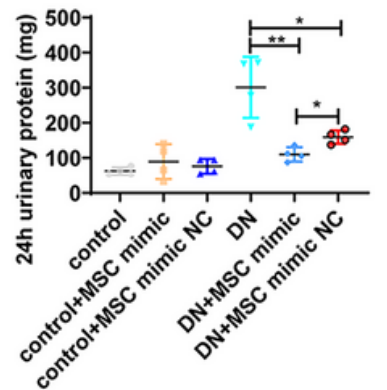

Kidney tissue e

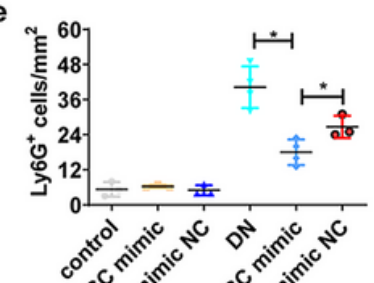

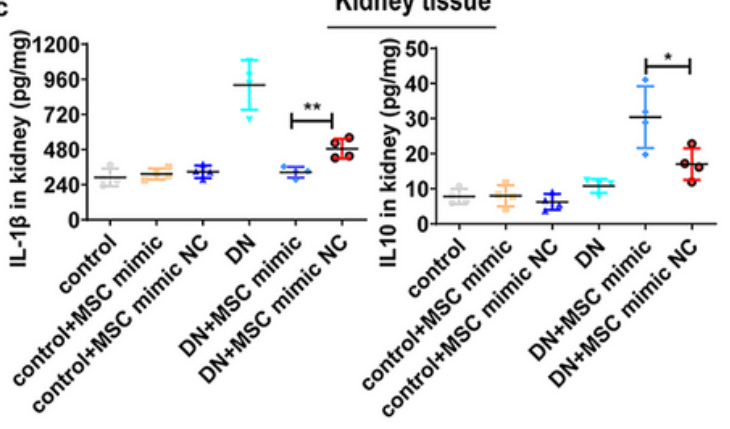
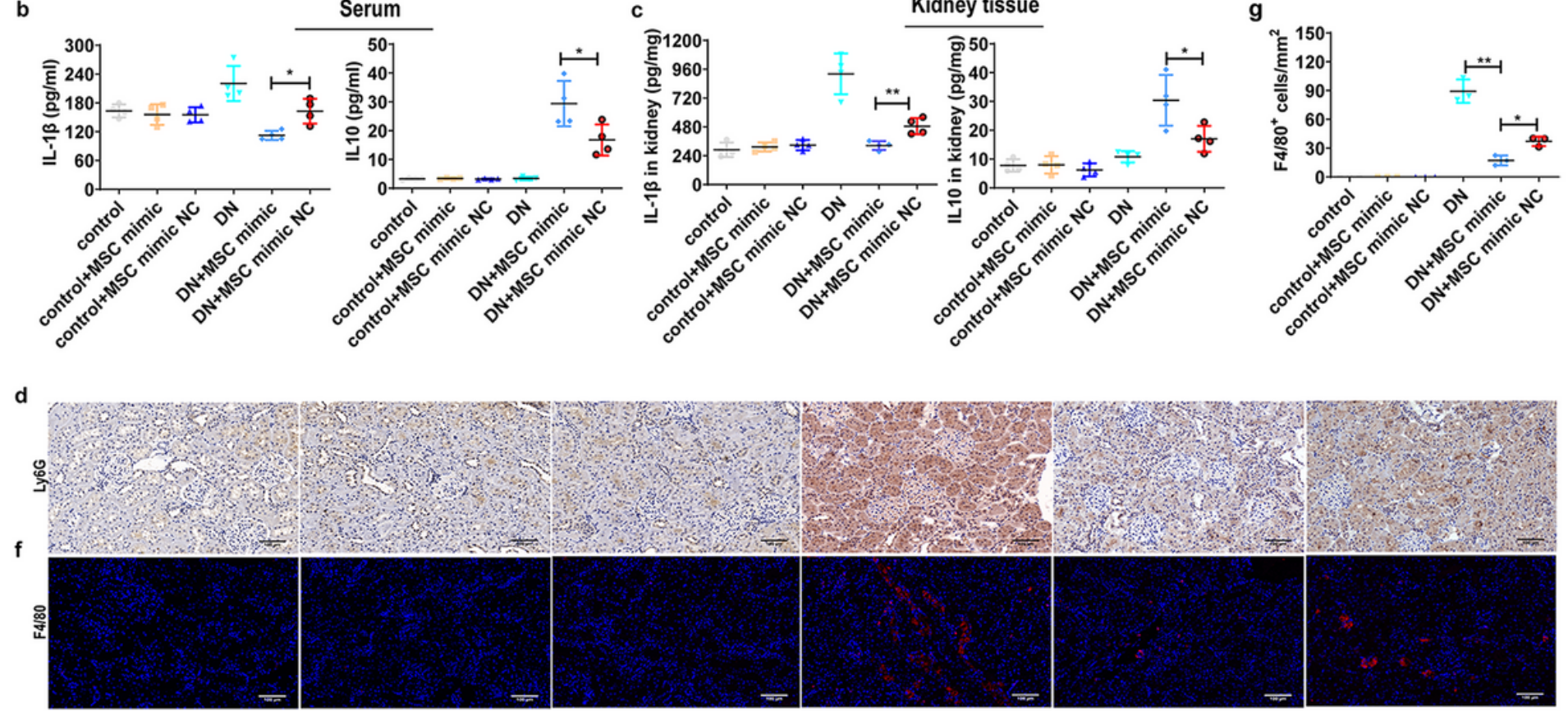

Figure 7

miR-146a-5p-modified UC-MSCs enhance the efficacy of anti-inflammation and renal function improvement in DN rats. UC-MSCs and miR-146a-5p-modified UC-MSCs were intravenously administrated to explore the renal protection and macrophage polarization regulation of miR-146a-5p on DN rats. a Renal function was assessed by measuring serum creatinine, serum urea nitrogen, $24 \mathrm{~h}$ total urine protein and creatinine clearance rate in rats. b, c Concentration of IL-1 $\beta$ and IL-10 in the kidney and serum from each group of rats as measured by ELISA, $n=4$ rats/group. $d$, e Infiltrated neutrophils in the kidney as represented by Ly6G immunohistochemistry staining. $\mathrm{f}, \mathrm{g}$ Infiltrated macrophages in the kidney as represented by $\mathrm{F} 4 / 80$ immunofluorescence staining, $\mathrm{n}=3$ rats/group, Scale bar: $100 \mu \mathrm{m}$. 
a
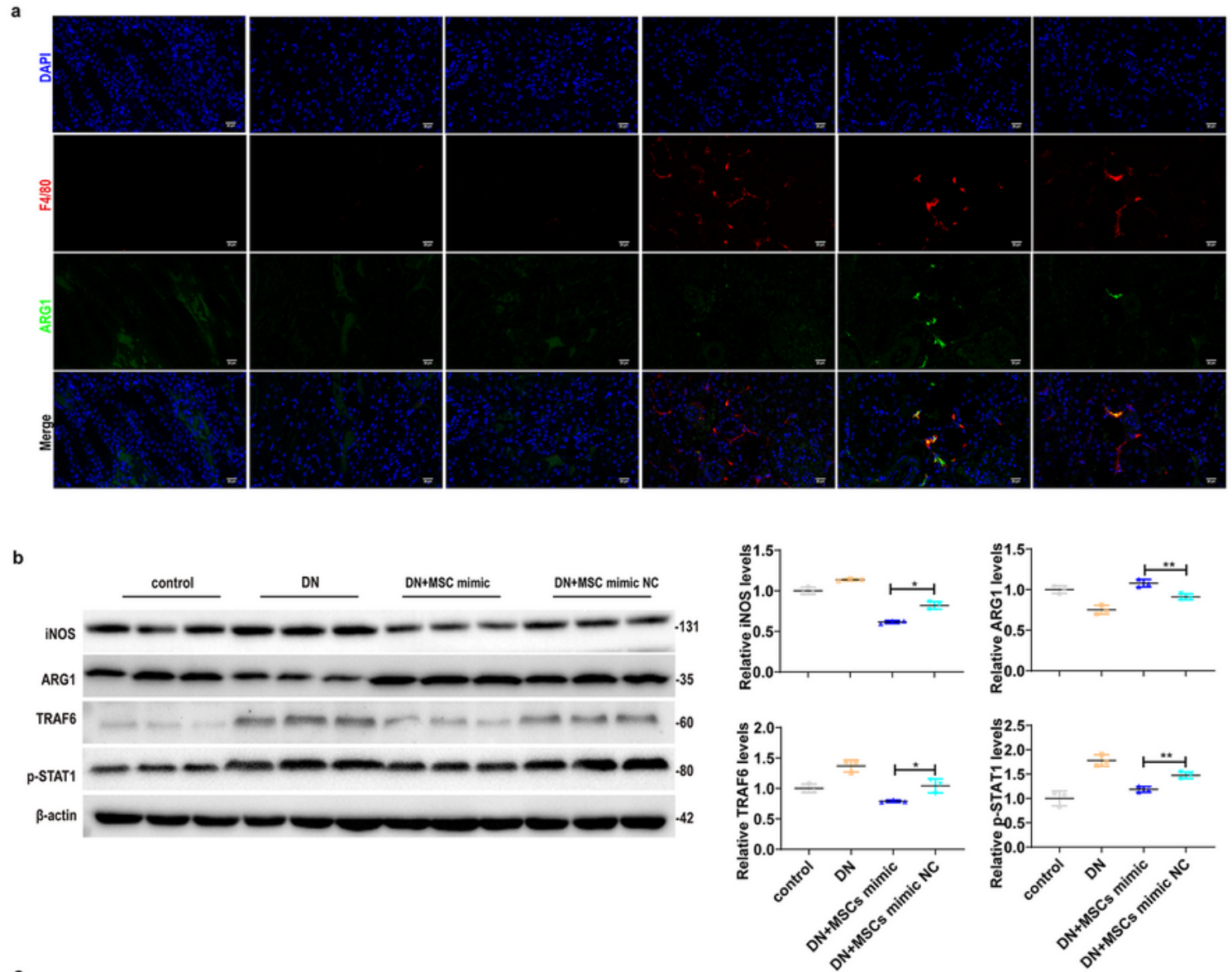

c

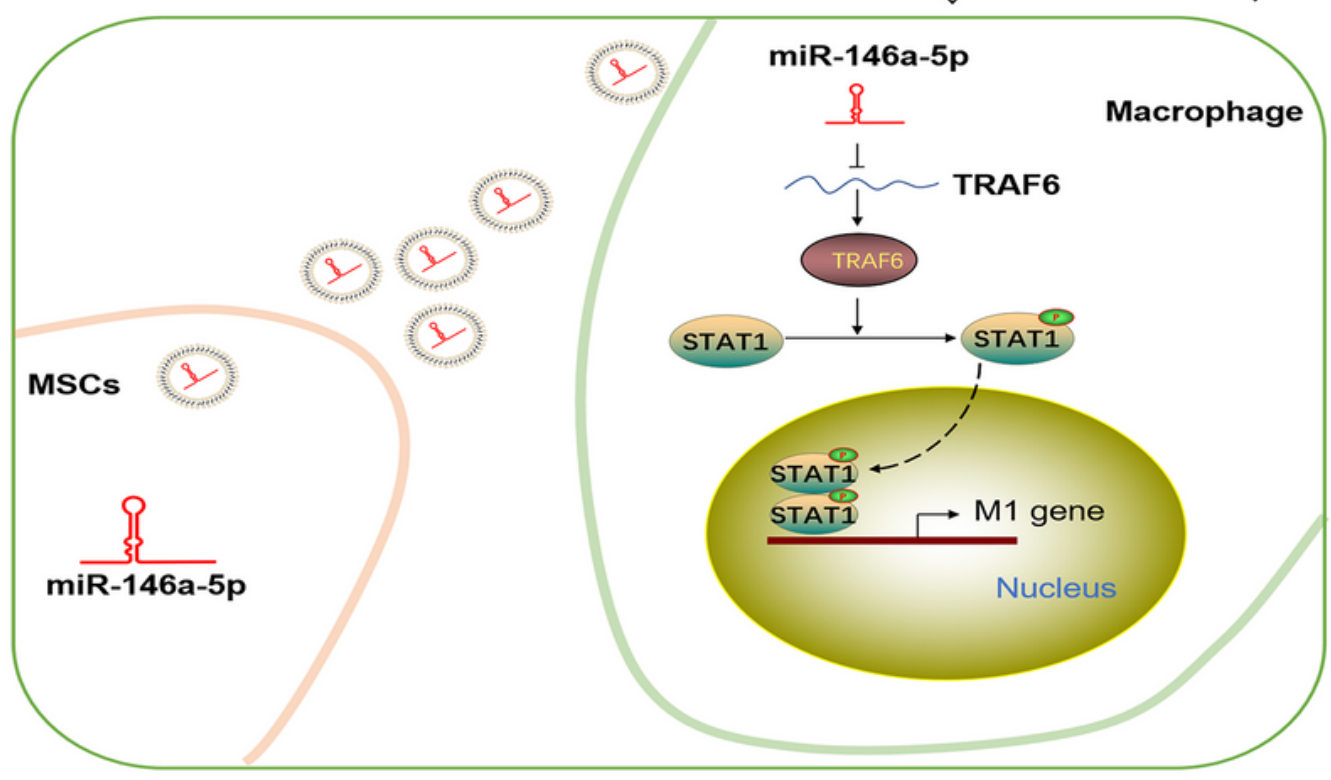

\section{Figure 8}

miR-146a-5p-modified UC-MSCs facilitate M2 macrophage polarization in DN rats. a Representative immunofluorescence staining for the macrophage marker F4/80 (red) and ARG1 (green) colocalization in the kidney, Scale bar: $20 \mu \mathrm{m}$. b Relative protein expression and semi-quantitative analysis of iNOS, ARG1, TRAF6, and p-STAT1 in the kidney. Data presented as mean \pm SD. c UC-MSCs, overexpressing miR-146a$5 p$, can be delivered to damaged kidneys to inhibit M1 macrophage activation but facilitate M2 
macrophage polarization by targeting TRAF6-STAT1 signaling pathway, leading to reduced inflammation and renal injury in DN rats. Data presented as mean \pm SD of individuals included in each group. $p$ values were calculated using Student's t-test. ${ }^{*} P<0.05,{ }^{* \star} P<0.01$.

\section{Supplementary Files}

This is a list of supplementary files associated with this preprint. Click to download.

- supplementarymeterials.pdf 